\title{
Competitive Equilibria in Matching Models with Financial Constraints
}

Citation for published version (APA):

Herings, P. J-J., \& Zhou, Y. (2019). Competitive Equilibria in Matching Models with Financial Constraints. Maastricht University, Graduate School of Business and Economics. GSBE Research Memoranda No. 007 https://doi.org/10.26481/umagsb.2019007

Document status and date:

Published: 01/04/2019

DOI:

10.26481/umagsb.2019007

Document Version:

Publisher's PDF, also known as Version of record

\section{Please check the document version of this publication:}

- A submitted manuscript is the version of the article upon submission and before peer-review. There can be important differences between the submitted version and the official published version of record.

People interested in the research are advised to contact the author for the final version of the publication, or visit the DOI to the publisher's website.

- The final author version and the galley proof are versions of the publication after peer review.

- The final published version features the final layout of the paper including the volume, issue and page numbers.

Link to publication

\footnotetext{
General rights rights.

- You may freely distribute the URL identifying the publication in the public portal. please follow below link for the End User Agreement:

www.umlib.nl/taverne-license

Take down policy

If you believe that this document breaches copyright please contact us at:

repository@maastrichtuniversity.nl

providing details and we will investigate your claim.
}

Copyright and moral rights for the publications made accessible in the public portal are retained by the authors and/or other copyright owners and it is a condition of accessing publications that users recognise and abide by the legal requirements associated with these

- Users may download and print one copy of any publication from the public portal for the purpose of private study or research.

- You may not further distribute the material or use it for any profit-making activity or commercial gain

If the publication is distributed under the terms of Article $25 \mathrm{fa}$ of the Dutch Copyright Act, indicated by the "Taverne" license above, 


\section{Maastricht University}

P. Jean-Jacques Herings, Yu Zhou

Competitive Equilibria in Matching Models with Financial Constraints

RM/19/007

\section{GSBE}

Maastricht University School of Business and Economics

Graduate School of Business and Economics

P.O Box 616

NL-6200 MD Maastricht

The Netherlands 


\title{
Competitive Equilibria in Matching Models with Financial Constraints*
}

\author{
P. Jean-Jacques Herings ${ }^{\dagger} \quad \mathrm{Yu}_{\mathrm{Zhou}}^{\ddagger}$
}

March 20, 2019

\begin{abstract}
We consider the one-to-one two-sided matching with contracts model in which buyers face financial constraints. In this model there is a stable outcome, but not necessarily a competitive equilibrium as defined in the standard way. We propose a new equilibrium notion, quantity-constrained competitive equilibrium (QCCE) that allows buyers to form rational expectations on the lack of supply when their financial constraints are binding. We show the existence of QCCEs and establish the equivalence among QCCE outcomes, stable outcomes, and core outcomes. We also analyze the existence of QCCEs with uniform prices, the lattice property of QCCEs, and the rural hospital theorem of QCCEs. We finally examine the relation between models with financial constraints and models with price controls.
\end{abstract}

Keywords: Financial constraints, matching with contracts, stable outcome, quantityconstrained competitive equilibrium, equivalence result, lattice property, core outcome, rural hospital theorem.

JEL Classification: C71, C78, D45, D52.

*Zhou gratefully acknowledges financial support from Waseda Grant for Special Research Projects (2018S-214) and Grant-in-aid for Research Activity, Japan Society for the Promotion of Science (17H06828).

${ }^{\dagger}$ Department of Economics, Maastricht University, P.O. Box 616, 6200 MD, Maastricht, the Netherlands. E-mail: P.Herings@maastrichtuniversity.nl

${ }_{\ddagger}^{\ddagger}$ Main address: Waseda Institute for Advanced Study, Waseda University, 1-6-1 Nishi Waseda, Shinjukuku, Tokyo 169-8050, Japan. E-mail: zhouyu_0105@hotmail.com; Affiliated address: Institute of Social and Economic Research, Osaka University, 6-1 Mihogaoka, Ibaraki, Osaka 567-0047, Japan. 


\section{Introduction}

Standard models in matching and auction theory assume that agents face no financial constraints, i.e., they have neither liquidity nor credit constraints. However, financial constraints arise commonly, in a variety of situations, for example, in privatization processes in developing countries, in the spectrum licence auctions in European countries, and mergers and acquisitions amongst corporations. ${ }^{1}$

The analysis of models with financial constraints is qualitatively different from that of models without financial constraints. ${ }^{2}$ Financial constraints evidently influence agents' trading behaviors and shrink the number of potential transactions. Moreover, financial constraints may result in the lack of existence of competitive equilibria (Talman and Yang, 2015; van der Laan and Yang, 2016). In this paper, we argue that the standard definition of competitive equilibrium should be adjusted.

We study the one-to-one two-sided matching with contracts model in the spirit of Crawford and Knoer (1981), Roth (1984), and Hatfield and Milgrom (2005) in the presence of financial constraints. Buyers and sellers have the possibility to choose from a finite set of possible trades. Each trade is bilateral and designates its buyer and seller. A contract consists of a trade and a financial payment from the buyer to the seller for that trade. We allow that the payment changes continuously, so the set of contracts is infinite.

The utility functions of buyers and sellers are defined on the set of contracts. Utility functions satisfy standard continuity and monotonicity assumptions and need not be quasilinear. We introduce financial constraints on the buyers side. Any contract violating these constraints is not feasible. Our modeling of financial constraints is sufficiently general to allow for absence of such constraints as a special case and to allow constraints to be trade-dependent. As an example of the latter, for trades like houses, which can serve as collateral, distinct houses typically entail different financial constraints.

In our model, a stable outcome always exists, where stability is as defined in the standard way in the matching literature, see, e.g., Demange and Gale (1985), Roth and Sotomayor (1990), and Hatfield and Milgrom (2005). On the other hand, a competitive equilibrium may fail to exist. We argue that the standard definition of competitive equilibrium should be extended by a vector of trade-dependent quantity constraints. We allow buyers to form rational expectations on the lack of supply when their financial constraints are binding. In case the buyer expects that the seller is not willing to supply the trade, the buyer faces a binding quantity constraint, and the trade is excluded from the buyer's

\footnotetext{
${ }^{1}$ See, e.g., Maskin (2000), Illing and Klüh (2003), and Liao (2014) for details.

${ }^{2}$ See, e.g., Che and Gale (1998), Benoit and Krishna (2001), Ausubel and Milgrom (2002), Dobzinski, Lavi, and Nisan (2012), Talman and Yang (2015), and van der Laan and Yang (2016) for the analysis of auction models with financial constraints.
} 
budget set. Otherwise, the buyer is free to demand this trade. Sellers are not subject to quantity constraints. The resulting equilibrium notion is called quantity-constrained competitive equilibrium (QCCE). A QCCE consists of a price vector assigning a price to each trade, a vector of quantity constraints assigning a quantity constraint to each trade, and an outcome. Quantity constraints are only allowed to be binding when a buyer's financial constraint is binding. The outcome consists of utility maximizing contracts for the buyers and the sellers at the given prices and quantity constraints. When financial constraints are absent or not binding, a QCCE is just a competitive equilibrium.

We first show the existence of a QCCE. The proof makes use of the existence of a stable outcome. Given a stable outcome, we find prices and quantity constraints which together constitute a QCCE. We also show the reverse, i.e., that each QCCE outcome is a stable outcome, which establishes an equivalence result. We further establish the equivalence between core outcomes and the set of QCCE outcomes.

A QCCE may involve personalized prices, where the seller charges different prices to different buyers of the same object for instance. We investigate the existence of QCCEs with uniform prices. We present three sets of sufficient conditions to obtain QCCEs with seller-uniform prices, buyer-uniform prices, and uniform prices, respectively.

We analyze the structure of QCCE prices and agents' utilities and show that the lattice property does not hold. However, if we consider the QCCEs compatible with a given vector of quantity constraints, then the lattice property is preserved. Due to the financial constraints, the completeness of the lattice may fail to hold. We also show that the rural hospital theorem fails to hold for QCCEs. However, for QCCEs compatible with a given vector of quantity constraints, a version of the rural hospital theorem can be shown.

Finally, we relate QCCEs of models with financial constraints to Drèze equilibria in general equilibrium models with price rigidities, see Bénassy (1975), Drèze (1975), Younès (1975), van der Laan (1980), and Herings (1996, 2018).

The connections among stable outcomes, core outcomes, and competitive equilibria have been studied for various models. Shapley and Shubik (1971) show the equivalence between the set of stable payoffs and the set of competitive equilibrium payoffs in the assignment game when agents have quasi-linear utility functions. Quinzii (1984) establishes the same result in a one-to-one multi-sided matching model and allows agents to have general utility functions. Kojima, Sun, and Yu (2018) discuss the equivalence between the set of core outcomes and the set of competitive equilibria in a many-to-one matching model when agents have general utility functions, but are subject to interval constraints. In models of trading networks, the equivalence between the set of outcomes induced by competitive equilibria and the set of stable outcomes also holds, see Hatfield, Kominers, Nichifor, Ostrovsky, and Westkamp (2013), Candogan, Epitropou, and Vohra (2017), and Fleiner, 
Janko, Tamura, and Teytelboym (2018). Herings (2018) studies models where prices are subject to price controls, the marriage model of Gale and Shapley (1962) being a special case, and establishes the equivalence between the set of stable outcomes and the set of Drèze equilibrium outcomes. All these equivalence results are derived in settings without financial constraints.

In models of trading networks without financial constraints, Hatfield et al. (2013), Candogan et al. (2017), and Schlegel (2019) provide sufficient conditions to guarantee that an arbitrary competitive equilibrium can be transformed into a competitive equilibrium with uniform prices by making suitable price adjustments. Different from these three works, when financial constraints are introduced, additional conditions on the financial constraints are needed to derive a QCCE with uniform prices from an arbitrary QCCE. Moreover, on top of price adjustments, also changes in the quantity constraints are needed.

The lattice property of competitive equilibrium payoffs and prices is intensively investigated in various directions, like matching models, e.g., Shapley and Shubik (1971), Demange and Gale (1985), Hatfield and Milgrom (2005), Hatfield and Kojima (2010), Kucuksenel (2011), and Kojima et al. (2018), and models of trading networks, e.g., Ostrovsky (2008), Hatfield and Kominers (2012), Hatfield et al. (2013), Candogan et al. (2017), Fleiner et al. (2018), and Schlegel (2019). When financial constraints are introduced, the lattice property of prices and agents' utilities in QCCEs fails to hold.

The rural hospital theorem of stable matchings and competitive equilibria has been established in different models, like matching models, e.g., Roth (1986), Demange and Gale (1985), Hatfield and Milgrom (2005), Hatfield and Kojima (2010), and Kojima et al. (2018), and models of trading networks, e.g., Hatfield and Kominers (2012), Fleiner et al. (2018), and Schlegel (2019). It shows the invariance of the sets of matched agents in different stable matchings and competitive equilibria. In the presence of financial constraints, the rural hospital theorem fails to hold for QCCEs.

Talman and Yang (2015) study the assignment model with financial constraints. There is a single seller owning all the items and charging a uniform price for each item. Each buyer wants to obtain at most one item. Talman and Yang (2015) constructively show the existence of a core allocation. Since there is only one seller, the set of core allocations is strictly larger than the set of competitive equilibria or the set of QCCEs. Van der Laan and Yang (2016) propose an equilibrium under allotment. The outcome induced by the equilibrium under allotment may not be in the core. Van der Laan, Talman, and Yang (2018) propose a price adjustment process that either finds a minimum price competitive equilibrium in a finite number of steps or exclusively validates the non-existence of competitive equilibrium.

The paper is organized as follows. Section 2 introduces the model and Section 3 presents 
the notion of stable outcome. Section 4 introduces QCCEs and shows their existence. Section 5 studies the relations among QCCE outcomes, stable outcomes, and core outcomes, QCCEs with uniform prices, the lattice property of QCCEs, and the rural hospital theorem of QCCEs. Section 6 discusses the relation between models with financial constraints and those with price controls. Section 7 contains the concluding remarks.

\section{The Matching Model with Financial Constraints}

There is a finite set of buyers $B$ and a finite set of sellers $S$. Buyers and sellers can participate in bilateral trades in a finite set $T$. Each trade $t \in T$ is associated with a buyer $\beta(t) \in B$ and a seller $\sigma(t) \in S$. A trade corresponds to the precise contractual terms of the delivery of a good or service from the seller to the buyer, but leaving out the price against which this transaction occurs. We denote the sets of trades in $T$ involving buyer $b$ and seller $s$ by

$$
\begin{aligned}
& T^{b}=\{t \in T \mid \beta(t)=b\}, \\
& T^{s}=\{t \in T \mid \sigma(t)=s\} .
\end{aligned}
$$

Transactions take place by signing contracts. Each contract $c=(t, m) \in T \times \mathbb{R}$ specifies a trade $\tau(c)=t \in T$ and a payment $\mu(c)=m \in \mathbb{R}$, where the payment corresponds to the amount of money that is transferred from buyer $\beta(t)$ to seller $\sigma(t)$. A negative payment means that signing the contract requires a compensation from the seller to the buyer. We consider a one-to-one matching set-up: A buyer signs a contract with at most one seller and a seller signs a contract with at most one buyer. Each buyer and seller can choose the no-trade option, denoted by $\emptyset$.

For each buyer $b \in B$, the utility function $u^{b}:\left(T^{b} \times \mathbb{R}\right) \cup\{\emptyset\} \rightarrow \mathbb{R}$ is such that (b-i) for each $t \in T^{b}, u^{b}(t, \cdot)$ is continuous and strictly decreasing in $\mathbb{R}$, and (b-ii) for each $t \in T^{b}$, there is $m \in \mathbb{R}$ such that $u^{b}(t, m) \leq u^{b}(\emptyset)$. Condition (b-i) says that given a trade, a lower payment improves the buyer's utility. Condition (b-ii) says that there is a certain monetary limit such that a buyer will not trade when the payment is above that limit.

For each seller $s \in S$, the utility function $u^{s}:\left(T^{s} \times \mathbb{R}\right) \cup\{\emptyset\} \rightarrow \mathbb{R}$ is such that (s-i) for each $t \in T^{s}, u^{s}(t, \cdot)$ is continuous and strictly increasing in $\mathbb{R}$, and (s-ii) for each $t \in T^{s}$, there is $m \in \mathbb{R}$ such that $u^{s}(t, m) \leq u^{s}(\emptyset)$. Condition (s-i) says that given a trade, a higher payment improves the seller's utility. Condition (s-ii) says that there is a certain monetary limit such that the seller will not trade when the payment is below that limit.

Conditions (b-i) and (s-i) allow for the case where a trade $t \in T^{i}$ is better than a trade $t^{\prime} \in T^{i}$ for agent $i \in B \cup S$, irrespective of the amount of money involved: For every $m, m^{\prime} \in \mathbb{R}, u^{i}(t, m)>u^{i}\left(t^{\prime}, m^{\prime}\right)$. Let $u=\left(u^{i}\right)_{i \in B \cup S}$ be the profile of utility functions. 
Buyers may face both liquidity and credit constraints, which we refer to as financial constraints in the sequel. In case of financial constraints, there is a maximum amount of money that buyers can use to make payments. For every buyer $b \in B$, for every trade $t \in T^{b}$, the corresponding financial constraint is denoted by $l_{t}^{b} \in \overline{\mathbb{R}}_{+}=\mathbb{R}_{+} \cup\{+\infty\}$. If $l_{t}^{b}=+\infty$, then there is no financial constraint for buyer $b$ when making the trade $t$. We use the notation $l^{b}=\left(l_{t}^{b}\right)_{t \in T^{b}}$ and $l=\left(l^{b}\right)_{b \in B}$.

The primitives of the economy are summarized by $\mathcal{E}=(B, S, T, u, l)$.

Our model of matching with contracts and financial constraints includes the two important models below as special cases.

Example 2.1: The matching model without financial constraints, e.g., Crawford and Knoer (1981), Demange and Gale (1985), and Alkan and Gale (1990).

There are finite sets $B$ and $S$ of agents who want to match with one another. Agents that match can make use of monetary transfers. An agent $i \in B \cup S$ has a stand-alone utility $r_{i}$ of being unmatched. The matchings of agents in $B$ and $S$ can be modeled by the set of trades $T=B \times S$. Being unmatched corresponds to the no-trade option $\emptyset$. Utility functions are general and satisfy assumptions (b-i), (b-ii), (s-i), and (s-ii), together with an assumption of "finiteness," i.e., for each $i \in B \cup S$ and each $t \in T^{i}$, the range of $u^{i}(t, \cdot)$ is all of $\mathbb{R}$. The stand-alone value of an agent $i \in B \cup S$ is defined as $r_{i}=u^{i}(\emptyset)$. Since agents do not face financial constraints, we have $l_{t}^{b}=+\infty$ for each $b \in B$ and for each $t \in T^{b}$.

Example 2.2: The assignment model with financial constraints, e.g., Talman and Yang (2015), van der Laan and Yang (2016), and van der Laan et al. (2018).

There is a finite set of buyers $B$ and a finite set of sellers $S$. Each seller owns one item and is only willing to sell if the price is above a positive reserve price. A buyer is interested in at most one item. Buyers have financial constraints, which are independent of the items to be bought. Buyers have quasi-linear utility functions. As in Example 2.1, we can take the set of trades equal to $T=B \times S$. For every $b \in B, u^{b}(\emptyset)=0$ and, for every $t \in T^{b}, u^{b}(t, m)=u^{b}(t, 0)-m$. Since financial constraints do not depend on the item bought, we have, for every $t, t^{\prime} \in T^{b}, l_{t}^{b}=l_{t^{\prime}}^{b}$. For every seller $s \in S, u^{s}(\emptyset)>0$ denotes the reserve price for the item in the possession of seller $s$ and, for every $t, t^{\prime} \in T^{s}$, it holds that $u^{s}(t, \cdot)=u^{s}\left(t^{\prime}, \cdot\right)$, so the identity of the buyer does not matter for the seller.

\section{$3 \quad$ Stable Outcomes}

In this section, we define the standard notions of stable and strongly stable outcomes, see, e.g., Demange and Gale (1985), Roth and Sotomayor (1990), and Hatfield and Milgrom 
(2005). We show that a stable outcome exists. We present an example to show that a strongly stable outcome may not exist.

Given a set of contracts $Y \subseteq T \times \mathbb{R}, Y^{b}$ denotes the set of contracts involving buyer $b$ and $Y^{s}$ denotes the set of contracts involving seller $s$, so

$$
\begin{aligned}
& Y^{b}=\{c \in Y \mid \beta(\tau(c))=b\}, \\
& Y^{s}=\{c \in Y \mid \sigma(\tau(c))=s\} .
\end{aligned}
$$

Notice that in case there is no contract in $Y$ involving an agent $i \in B \cup S$, then $Y^{i}=\emptyset$.

Denote the set of feasible contracts by $\bar{Y}$, i.e.,

$$
\bar{Y}=\left\{(t, m) \in T \times \mathbb{R} \mid m \leq l_{t}^{\beta(t)}\right\} .
$$

The payment as specified in a feasible contract is compatible with the buyer's financial constraint.

A set of contracts $A \subseteq \bar{Y}$ is an outcome if for each $i \in B \cup S,\left|A^{i}\right| \leq 1$, so an outcome is a set of feasible contracts such that each agent is involved in at most one contract. Let $\mathcal{A}$ be the collection of outcomes, i.e.,

$$
\mathcal{A}=\left\{A \subseteq \bar{Y} \mid \text { for each } i \in B \cup S,\left|A^{i}\right| \leq 1\right\}
$$

Notice that $\emptyset \in \mathcal{A}$. For each $i \in B \cup S$, let $\mathcal{A}^{i}$ be the collection of singletons involving a contract for agent $i$ together with the no-trade option $\emptyset$, i.e.,

$$
\mathcal{A}^{i}=\{A \in \mathcal{A} \mid \text { for every } c \in A, i \in \beta(\tau(c)) \cup \sigma(\tau(c))\} \text {. }
$$

For every $i \in B \cup S$, the utility function $u^{i}$ over contracts induces the utility function $U^{i}$ over outcomes, for every $A \in \mathcal{A}$,

$$
U^{i}(A)= \begin{cases}u^{i}(\emptyset), & \text { if } A^{i}=\emptyset, \\ u^{i}(c), & \text { if } A^{i}=\{c\} .\end{cases}
$$

For each $i \in B \cup S$, for each $Y \subseteq \bar{Y}$, the set of optimal choices of agent $i$ within the set of contracts $Y$ is defined as

$$
\operatorname{Ch}^{i}(Y)=\underset{\left\{A^{i} \in \mathcal{A}^{i} \mid A^{i} \subseteq Y^{i}\right\}}{\arg \max } U^{i}\left(A^{i}\right)
$$

In case $Y$ is finite, it holds that $\operatorname{Ch}^{i}(Y)$ is non-empty. Notice that $\operatorname{Ch}^{i}(Y)=\{\emptyset\}$ means that the no-trade option is the optimal choice in $Y$ for agent $i$.

Definition 3.1: An outcome $A \in \mathcal{A}$ is stable if:

(i) For each $i \in B \cup S, A^{i} \in \mathrm{Ch}^{i}(A)$. 
(ii) There is no $c=(t, m) \in \bar{Y}$ such that $U^{\beta(t)}(\{c\})>U^{\beta(t)}(A)$ and $U^{\sigma(t)}(\{c\})>U^{\sigma(t)}(A)$.

A stable outcome $A$ is a set of feasible contracts, containing at most one contract for each agent. Condition (i) of Definition 3.1 corresponds to individual rationality. Agent $i$ is involved in a contract which is at least as good as no trade. Condition (ii) of Definition 3.1 corresponds to the absence of a blocking pair. There are no two agents who can sign a contract that makes both of them better off.

The basic idea of showing the existence of a stable outcome is as follows. First, we discretize the payments of contracts in $\bar{Y}$ and remove contracts that are worse than the no-trade option, leading to a finite set of feasible contracts with discretized payments $\bar{Y}^{\varepsilon}$. Then we use an adjustment process in the spirit of Gale and Shapley (1962), Crawford and Knoer (1981), Kelso and Crawford (1982), Demange, Gale, and Sotomayor (1986), and Hatfield and Milgrom (2005) that ends with a stable outcome for the set $\bar{Y}^{\varepsilon}$. Finally, we can use a limit argument to show that there is a stable outcome in $\bar{Y}$.

For every $b \in B$, for every $t \in T^{b}$, let $m_{t}^{b} \in \mathbb{R}$ be such that $u^{b}\left(t, m_{t}^{b}\right) \leq u^{b}(\emptyset)$ and $\bar{m}_{t}^{b}=\min \left\{l_{t}^{b}, m_{t}^{b}\right\}$. For every $s \in S$, for every $t \in T^{s}$, let $\underline{m}_{t}^{s} \in \mathbb{R}$ be such that $\underline{m}_{t}^{s} \leq \bar{m}_{t}^{\beta(t)}$ and $u^{s}\left(t, \underline{m}_{t}^{s}\right) \leq u^{s}(\emptyset)$. For each $\varepsilon>0$, the finite set of contracts $\bar{Y}^{\varepsilon}$ is defined by

$$
\bar{Y}^{\varepsilon}=\left\{(t, m) \in \bar{Y} \mid \exists k \in \mathbb{Z}^{+} \text {such that } m=\min \left\{\underline{m}_{t}^{\sigma(t)}+k \varepsilon, \bar{m}_{t}^{\beta(t)}\right\}\right\} .
$$

We now present an adjustment process to show that there is a stable outcome for the set of feasible contracts $\bar{Y}^{\varepsilon}$.

Definition 3.2: Let some $\varepsilon>0$ be given. The adjustment process consists of the following steps.

Step 0: Define $Y^{0}=\bar{Y}^{\varepsilon}$ and $A^{0}=\emptyset$. Each buyer $b \in B$ chooses one element $Z^{0 b} \in \mathrm{Ch}^{b}\left(Y^{0}\right)$. Define $Z^{0}=\cup_{b \in B} Z^{0 b}$. Each seller $s \in \sigma\left(Z^{0}\right)$ tentatively accepts one element $A^{1 s} \in \mathrm{Ch}^{s}\left(Z^{0}\right)$. Define $A^{1}=\cup_{s \in S} A^{1 s}$. If $A^{1}=Z^{0}$, then the process terminates and the contracts in $A^{1}$ are permanently accepted. Otherwise, go to Step 1.

Step $k(\geq 1)$ : Define $Y^{k}=Y^{k-1} \backslash\left(Z^{k-1} \backslash A^{k}\right)$. Each buyer $b \in B \backslash \beta\left(A^{k}\right)$ chooses one element $Z^{k b} \in \mathrm{Ch}^{b}\left(Y^{k}\right)$. Define $Z^{k}=A^{k} \cup\left(\cup_{b \in B \backslash \beta\left(A^{k}\right)} Z^{k b}\right)$. Each seller $s \in \sigma\left(Z^{k}\right)$ tentatively accepts one element $A^{(k+1) s} \in \mathrm{Ch}^{s}\left(Z^{k}\right)$. Define $A^{k+1}=\cup_{s \in S} A^{(k+1) s}$. If $A^{k+1}=Z^{k}$, then the process terminates and the contracts in $A^{k+1}$ are permanently accepted. Otherwise, go to Step $k+1$.

Lemma 3.3: For each $\varepsilon>0$, the adjustment process terminates in a finite number of steps with a set of permanently accepted contracts. This set constitutes a stable outcome for $\bar{Y}^{\varepsilon}$. 
Results that are closely related to Lemma 3.3 have been shown by, e.g., Crawford and Knoer (1981) and Kelso and Crawford (1982) in setting without financial constraints. For completeness, we provide a formal proof of Lemma 3.3 in Appendix A.1.

Now we state the existence result.

Proposition 3.4: Consider an economy $\mathcal{E}=(B, S, T, u, l)$. Then a stable outcome exists.

Proof: See Appendix A.2.

Q.E.D.

Alkan and Gale (1990) establish the existence of stable outcome in the matching model without financial constraints, as described in Example 2.1. That result is a by-product of Proposition 3.4.

We next give the definition of a strongly stable outcome.

Definition 3.5: An outcome $A \in \mathcal{A}$ is strongly stable if:

(i) For each $i \in B \cup S, A^{i} \in \mathrm{Ch}^{i}(A)$.

(ii) There is no $c=(t, m) \in \bar{Y}$ such that $U^{\beta(t)}(\{c\}) \geq U^{\beta(t)}(A)$ and $U^{\sigma(t)}(\{c\}) \geq U^{\sigma(t)}(A)$, with at least one inequality being strict.

Condition (i) in Definition 3.5 coincides with Condition (i) in Definition 3.1. Condition (ii) in Definition 3.5 is stronger than Condition (ii) in Definition 3.1 since in Definition 3.5 it is sufficient that only one of the trading partners has a strict improvement for a pairwise deviation to occur.

In the one-to-one matching with contracts model, if there are no financial constraints, then a stable outcome is also strongly stable (Crawford and Knoer, 1981; Demange and Gale, 1985). As we will see, in the presence of financial constraints, a strongly stable outcome may not exist.

Example 3.6: Let $B=\left\{b_{1}, b_{2}\right\}, S=\left\{s_{1}\right\}$, and $T=\left\{t, t^{\prime}\right\}$, where $\beta(t)=b_{1}$ and $\beta\left(t^{\prime}\right)=b_{2}$. For each $i \in B \cup S$, let $u^{i}(\emptyset)=0$. Let $u^{b_{1}}(t, m)=3-m, u^{b_{2}}\left(t^{\prime}, m\right)=4-m$, and $u^{s_{1}}(t, m)=u^{s_{1}}\left(t^{\prime}, m\right)=m$. The financial constraints of the buyers are given by $l_{t}^{b_{1}}=2$ and $l_{t^{\prime}}^{b_{2}}=2$. The set of feasible contracts is then given by $\bar{Y}=\{(t, m) \in T \times \mathbb{R} \mid m \leq 2\}$. The collection of outcomes is $\mathcal{A}=\{\emptyset\} \cup\{\{(t, m)\} \mid m \leq 2\} \cup\left\{\left\{\left(t^{\prime}, m\right)\right\} \mid m \leq 2\right\}$.

By contradiction, suppose that the outcome $A \in \mathcal{A}$ is strongly stable.

First, we show that $A \neq \emptyset$. Suppose $A=\emptyset$. Consider $c=(t, 2) \in \bar{Y}$. Since $U^{b_{1}}(A)=$ $0<1=U^{b_{1}}(\{c\})$ and $U^{s_{1}}(A)=0<2=U^{s_{1}}(\{c\})$, Condition (ii) of Definition 3.5 is violated, contradicting that $A$ is a strongly stable outcome. Consequently, it holds that $A \neq \emptyset$. 
We have that $A \in\{\{(t, m)\} \mid m \leq 2\} \cup\left\{\left\{\left(t^{\prime}, m\right)\right\} \mid m \leq 2\right\}$. By individual rationality for the seller it follows that $A \in\{\{(t, m)\} \mid 0 \leq m \leq 2\} \cup\left\{\left\{\left(t^{\prime}, m\right)\right\} \mid 0 \leq m \leq 2\right\}$.

Suppose $A=\{(t, m)\}$ for some $m \in[0,2]$. Consider $c=\left(t^{\prime}, 2\right) \in \bar{Y}$. Since $U^{b_{2}}(A)=$ $0<2=U^{b_{2}}(\{c\})$ and $U^{s_{1}}(A)=m \leq 2=U^{s_{1}}(\{c\})$, Condition (ii) of Definition 3.5 is violated, contradicting that $A$ is a strongly stable outcome.

We have that $A=\left\{\left(t^{\prime}, m\right)\right\}$ for some $m \in[0,2]$. Consider $c=(t, 2) \in \bar{Y}$. Since $U^{b_{1}}(A)=0<1=U^{b_{1}}(\{c\})$ and $U^{s_{1}}(A)=m \leq 2=U^{s_{1}}(\{c\})$, Condition (ii) of Definition 3.5 is violated, contradicting that $A$ is a strongly stable outcome.

We have shown that there is no strongly stable outcome.

\section{Quantity-constrained Competitive Equilibrium}

In the competitive analysis of matching models with contracts and financial constraints, each trade $t \in T$ is assigned a price $p_{t} \in \mathbb{R}$, resulting in a price vector $p \in \mathbb{R}^{T}$. Prices are allowed to be personalized. For example, the price vector $p \in \mathbb{R}^{T}$ can be such that a seller $s \in S$ can sell the same physical commodity at different prices to different buyers. Personalized prices have been used before in competitive settings, see, e.g., Koopmans and Beckmann (1957), Makowski (1979), Hatfield et al. (2013), Candogan et al. (2017), and Fleiner et al. (2018).

In a competitive equilibrium, buyers and sellers take prices $p \in \mathbb{R}^{T}$ as given and choose optimal trades. We now argue that in the economy of Example 3.6, a competitive equilibrium does not exist. We argue first that there are no competitive equilibria without trade. The seller is only not willing to supply a trade if the price is less than or equal to zero, but then there is demand by both buyers. Since there is only one seller, a single unit will therefore be traded at a competitive equilibrium. To get a total demand of one unit, the price of one trade has to be above 2 and the price of the other trade should be less than or equal to 2 . The seller will then choose to supply the trade with a price above 2 , but for that trade there is no demand. This shows that there is no competitive equilibrium in the economy of Example 3.6.

The price system where both prices are equal to 2 does not correspond to a competitive equilibrium. The reason is that there is a total demand of two units at these prices, whereas the seller is only going to supply one unit. Nevertheless, we will argue that these prices should be regarded as equilibrium prices. We achieve this by extending the standard notion of competitive equilibrium by a vector of quantity constraints.

In case the financial constraint is binding for some trade, we allow the buyer to hold the expectation that there is no supply of such a trade by the seller and we refer to such an expectation as a quantity constraint. These expectations should be rational, so at equilibrium, if a buyer expects a quantity constraint on a trade, then there should indeed 
be no supply by the seller of that trade. For each $t \in T$, let $q_{t} \in\{0,1\}$ be the quantity constraint of trade $t$, where $q_{t}=0$ means that buyer $\beta(t)$ expects trade $t$ to be supplied and $q_{t}=1$ means that buyer $\beta(t)$ expects that trade $t$ is not available. We denote the vector of quantity constraints by $q=\left(q_{t}\right)_{t \in T}$.

The budget set of buyer $b \in B$ at $(p, q) \in \mathbb{R}^{T} \times\{0,1\}^{T}$ is given by

$$
\gamma^{b}(p, q)=\left\{\{c\} \in \mathcal{A} \mid \tau(c) \in T^{b}, \mu(c)=p_{\tau(c)} \text {, and } q_{\tau(c)}=0\right\} \cup\{\emptyset\} .
$$

The budget set of buyer $b$ is non-empty, since it always contains the no-trade option, $\emptyset$.

The demand set of buyer $b \in B$ at $(p, q) \in \mathbb{R}^{T} \times\{0,1\}^{T}$ is given by

$$
\delta^{b}(p, q)=\underset{A^{b} \in \gamma^{b}(p, q)}{\arg \max } U^{b}\left(A^{b}\right) .
$$

Since a seller does not face financial constraints, the seller's decision problem is the usual one. The budget set of seller $s \in S$ at $p \in \mathbb{R}^{T}$ is given by

$$
\gamma^{s}(p)=\left\{\{c\} \in \mathcal{A} \mid \tau(c) \in T^{s} \text { and } \mu(c)=p_{\tau(c)}\right\} \cup\{\emptyset\}
$$

The demand set of seller $s \in S$ at $p \in \mathbb{R}^{T}$ is given by

$$
\delta^{s}(p)=\underset{A^{s} \in \gamma^{s}(p)}{\arg \max } U^{s}\left(A^{s}\right) .
$$

We are now in a position to define our equilibrium concept.

Definition 4.1: An element $(p, q, A) \in \mathbb{R}^{T} \times\{0,1\}^{T} \times \mathcal{A}$ is a quantity-constrained competitive equilibrium ( $Q C C E$ ) for the economy $\mathcal{E}=(B, S, T, u, l)$ if:

(i) For each $b \in B, A^{b} \in \delta^{b}(p, q)$.

(ii) For each $s \in S, A^{s} \in \delta^{s}(p)$.

(iii) For each $t \in T$, if $p_{t}<l_{t}^{\beta(t)}$, then $q_{t}=0$.

The first two conditions correspond to standard optimization by buyers and sellers that take $p$ and $q$ as given. These two conditions also imply equality of demand and supply at a QCCE. The third condition reflects that when the price of trade $t$ is below buyer $\beta(t)$ 's financial constraint, then buyer $\beta(t)$ does not expect a quantity constraint on trade $t$. If, for every $t \in T, q_{t}=0$, then a QCCE reduces to a competitive equilibrium. It follows from Condition (iii) of Definition 4.1 that in models without financial constraints, i.e., for every $t \in T, l_{t}^{\beta(t)}=+\infty$, a QCCE reduces to a competitive equilibrium.

Consider a QCCE with $q_{t}=1$ for some $t \in T$. It holds that the expectations of buyer $\beta(t)$ are rational. Indeed, since $\left\{\left(t, p_{t}\right)\right\} \notin \gamma^{\beta(t)}(p, q)$, it holds that $\left(t, p_{t}\right)$ does not belong to the QCCE outcome $A$, so $A^{\sigma(t)}$ does not involve trade $t$, and trade $t$ is therefore not supplied by seller $\sigma(t)$. 
Example 4.2 illustrates the concept of QCCE for the economy of Example 3.6. It also presents the formal argument that a competitive equilibrium does not exist in the economy of Example 3.6.

Example 4.2: Consider the economy of Example 3.6. We show that there is no competitive equilibrium. Suppose, to derive a contradiction, that a competitive equilibrium exists or, equivalently, that there is a $\operatorname{QCCE}(p, q, A)$ with $q_{t}=q_{t^{\prime}}=0$. We have that $\delta^{b_{1}}(p, q)=$ $\left\{\left\{\left(t, p_{t}\right)\right\}\right\}$ if $p_{t} \leq 2$ and $\delta^{b_{2}}(p, q)=\left\{\left\{\left(t^{\prime}, p_{t^{\prime}}\right)\right\}\right\}$ if $p_{t^{\prime}} \leq 2$. Buyers therefore demand a total of two units if $\max \left\{p_{t}, p_{t^{\prime}}\right\} \leq 2$, which implies there cannot be equality of supply and demand. It follows that $\max \left\{p_{t}, p_{t^{\prime}}\right\}>2$. It also holds that $\delta^{b_{1}}(p, q)=\{\emptyset\}$ if $p_{t}>2$ and $\delta^{b_{2}}(p, q)=\{\emptyset\}$ if $p_{t^{\prime}}>2$. There is no demand if $\min \left\{p_{t}, p_{t^{\prime}}\right\}>2$, whereas supply equals one unit in this case, so again there is no equality of supply and demand. It follows that $\min \left\{p_{t}, p_{t^{\prime}}\right\} \leq 2$. Since $\min \left\{p_{t}, p_{t^{\prime}}\right\} \leq 2<\max \left\{p_{t}, p_{t^{\prime}}\right\}$, it holds that $p_{t} \neq p_{t^{\prime}}$ and only one of these prices exceeds 2 . The seller will supply the trade with the highest price, whereas the buyer involved in this trade will demand the no-trade option. We have obtained a contradiction. Consequently, a competitive equilibrium does not exist.

Let $(p, q, A)$ be such that $p=\left(p_{t}, p_{t^{\prime}}\right)=(2,2), q=\left(q_{t}, q_{t^{\prime}}\right)=(1,0)$, and $A=\left\{\left(t^{\prime}, 2\right)\right\}$. We show that $(p, q, A)$ is a $\mathrm{QCCE}$.

First, $(p, q, A)$ satisfies Conditions (i) and (ii) of Definition 4.1. For buyer $b_{1}$, it holds that $\gamma^{b_{1}}(p, q)=\{\emptyset\}$ and so $A^{b_{1}}=\emptyset \in \delta^{b_{1}}(p, q)=\{\emptyset\}$. For buyer $b_{2}$, it holds that $\gamma^{b_{2}}(p, q)=\left\{\emptyset,\left\{\left(t^{\prime}, 2\right)\right\}\right\}$ and $A^{b_{2}}=A=\left\{\left(t^{\prime}, 2\right)\right\} \in \delta^{b_{2}}(p, q)=\left\{\left\{\left(t^{\prime}, 2\right)\right\}\right\}$. For seller $s_{1}$, it holds that $\gamma^{s_{1}}(p)=\left\{\{(t, 2)\},\left\{\left(t^{\prime}, 2\right)\right\}, \emptyset\right\}$. We have that $A^{s_{1}}=A=\left\{\left(t^{\prime}, 2\right)\right\} \in \delta^{s_{1}}(p)=$ $\left\{\{(t, 2)\},\left\{\left(t^{\prime}, 2\right)\right\}\right\}$.

It is easily seen that $(p, q, A)$ satisfies Condition (iii) of Definition 4.1.

Obviously, a similar argument can be used to show that $\left(p^{\prime}, q^{\prime}, A^{\prime}\right)$ such that $p^{\prime}=$ $\left(p_{t}^{\prime}, p_{t^{\prime}}^{\prime}\right)=(2,2), q^{\prime}=\left(q_{t}^{\prime}, q_{t^{\prime}}^{\prime}\right)=(0,1)$, and $A^{\prime}=\{(t, 2)\}$ is a QCCE.

The following result provides the key to show the existence of a QCCE. It says that given a stable outcome $A$, we can always find a suitable price vector $p$ and a vector of quantity constraints $q$ such that $(p, q, A)$ is a $\mathrm{QCCE}$.

Proposition 4.3: Consider an economy $\mathcal{E}=(B, S, T, u, l)$. Let $A$ be a stable outcome of $\mathcal{E}$. Then there is a pair $(p, q) \in \mathbb{R}^{T} \times\{0,1\}^{T}$ such that $(p, q, A)$ is a $Q C C E$ for $\mathcal{E}$.

Proof: See Appendix A.3.

Q.E.D.

The sketch of the proof of Proposition 4.3 is as follows. For every buyer $b \in B$ we partition the set of trades $T^{b}$ into three classes. The first class consists of the trades such 
that at any payment, buyer $b$ prefers $A^{b}$ to those trades. A trade belongs to the second class if there is a payment satisfying the financial constraint of buyer $b$ for that trade and being such that buyer $b$ is indifferent between $A^{b}$ and that trade. The third class consists of the trades such that at any payment satisfying the financial constraints of buyer $b$, buyer $b$ strictly prefers those trades to $A^{b}$. For every seller $s \in S$, we divide the trades in $T^{s}$ into two classes. The first class consists of the trades such that at any payment, seller $s$ prefers $A^{s}$ to those trades. A trade belongs to the second class if there is a payment making seller $s$ indifferent between $A^{s}$ and that trade.

On the basis of these partitions, we assign to each trade a price and a quantity constraint. Each trade that is part of the stable outcome is assigned a price equal to the amount of money in the associated contract and a quantity constraint equal to zero. A quantity constraint is set equal to one if and only if a trade belongs to the third class for the buyer. A trade belonging to the second class of the seller is assigned a price equal to the payment that makes the seller indifferent. A trade belonging to the first class of the seller is assigned a price equal to the payment making the buyer not better off than at the stable outcome.

Propositions 3.4 and 4.3 imply the existence of a QCCE.

Corollary 4.4: Consider an economy $\mathcal{E}=(B, S, T, u, l)$. Then a QCCE exists.

\section{Properties of QCCEs}

In this section, we investigate the features of QCCEs in more detail. In turn, we study the equivalence between QCCE outcomes and stable outcomes, the equivalence between QCCE outcomes and core outcomes, the existence of QCCEs with uniform prices, the lattice property of QCCEs, and the rural hospital theorem of QCCEs.

\subsection{Equivalence between QCCE Outcomes and Stable Outcomes}

Shapley and Shubik (1971) consider the assignment game and show that the set of stable payoffs coincides with the set of competitive equilibrium payoffs. Such an equivalence relation is extended to capture one-to-one multi-sided matching by Quinzii (1984), trading networks by Hatfield et al. (2013), Candogan et al. (2017), and Fleiner et al. (2018), and one-to-one matching with price controls by Herings (2018). The following result establishes the equivalence in a setting with financial constraints.

For an economy $\mathcal{E}$, let

$$
\begin{aligned}
\mathcal{A}^{\mathrm{SO}}(\mathcal{E}) & =\{A \in \mathcal{A} \mid A \text { is a stable outcome }\} \\
\mathcal{A}^{\mathrm{QCCE}}(\mathcal{E}) & =\left\{A \in \mathcal{A} \mid \text { there is }(p, q) \in \mathbb{R}^{T} \times\{0,1\}^{T} \text { such that }(p, q, A) \text { is a QCCE }\right\}
\end{aligned}
$$


be the set of stable outcomes and the set of QCCE outcomes of $\mathcal{E}$, respectively.

Theorem 5.1 establishes the equivalence between $\mathcal{A}^{\mathrm{SO}}(\mathcal{E})$ and $\mathcal{A}^{\mathrm{QCCE}}(\mathcal{E})$.

Theorem 5.1: Consider an economy $\mathcal{E}=(B, S, T, u, l)$. Then $\mathcal{A}^{\mathrm{SO}}(\mathcal{E})=\mathcal{A}^{\mathrm{QCCE}}(\mathcal{E}) \neq \emptyset$.

Proof: See Appendix A.4.

Q.E.D.

Since our model contains the assignment model with financial constraints as a special case, the above equivalence result also holds in that model. In the assignment model with financial constraints, Definition 3.1 in van der Laan and Yang (2016) presents the notion of an equilibrium under allotment.

Definition 5.2: Let the economy $\mathcal{E}$ correspond to the assignment model with financial constraints, see Example 2.2. An element $(p, q, A) \in \mathbb{R}^{T} \times\{0,1\}^{T} \times \mathcal{A}$ is an equilibrium under allotment of $\mathcal{E}$ if:

(i) For each $b \in B, A^{b} \in \delta^{b}(p, q)$.

(ii) For each $s \in S, A^{s} \in \delta^{s}(p)$.

(iii) For each $t \in T$, if $q_{t}=1$, then $\left\{\left(t, p_{t}\right)\right\} \in \delta^{\beta(t)}\left(p,\left(q_{-t}, 0\right)\right)$ and there is $c^{\prime}=\left(t^{\prime}, m^{\prime}\right) \in A$ such that $\sigma\left(t^{\prime}\right)=\sigma(t), \beta\left(t^{\prime}\right) \neq \beta(t)$, and $p_{t^{\prime}}=m^{\prime}=l_{t^{\prime}}^{\beta\left(t^{\prime}\right)}$.

If a buyer expects a quantity constraint on an item in an equilibrium under allotment, then the seller is trading the item with another buyer whose financial constraint is binding. We now give an example such that the outcome of an equilibrium under allotment is not stable. This equilibrium notion is therefore qualitatively different from a QCCE.

Example 5.3: Let $B=\left\{b_{1}, b_{2}\right\}, S=\left\{s_{1}\right\}$, and $T=\left\{t, t^{\prime}\right\}$, where $\beta(t)=b_{1}$ and $\beta\left(t^{\prime}\right)=b_{2}$. For each $i \in B \cup S$, let $u^{i}(\emptyset)=0$. Let $u^{b_{1}}(t, m)=3-m, u^{b_{2}}\left(t^{\prime}, m\right)=4-m$, and $u^{s_{1}}(t, m)=u^{s_{1}}\left(t^{\prime}, m\right)=m$. The financial constraints of the buyers are given by $l_{t}^{b_{1}}=2$ and $l_{t^{\prime}}^{b_{2}}=3$. This is the same economy as in Example 3.6, except that the financial constraint of buyer $b_{2}$ has been raised from 2 to 3 . It is easily verified that the collection of stable outcomes is $\mathcal{A}^{\mathrm{SO}}(\mathcal{E})=\left\{\left\{\left(t^{\prime}, m\right)\right\} \mid 2 \leq m \leq 3\right\}$.

Let $p=\left(p_{t}, p_{t^{\prime}}\right)=(2,2), q=\left(q_{t}, q_{t^{\prime}}\right)=(0,1)$, and $A=\{(t, 2)\}$. It is not hard to see that $(p, q, A)$ is an equilibrium under allotment. However, $A \notin \mathcal{A}^{\mathrm{SO}}(\mathcal{E})$, i.e., $A$ is not a stable outcome.

\subsection{Equivalence between QCCE Outcomes and Core Outcomes}

In the following, we discuss the relation between QCCE outcomes and core outcomes in the presence of financial constraints. 
Definition 5.4: An outcome $A \in \mathcal{A}$ is a core outcome if there is no outcome $A^{\prime} \in \mathcal{A}$ and a set of agents $N \subseteq B \cup S$ such that:

(i) For each $i \in(B \cup S) \backslash N,\left(A^{\prime}\right)^{i}=\emptyset$.

(ii) For each $j \in N, U^{j}\left(A^{\prime}\right)>U^{j}(A)$.

Given an economy $\mathcal{E}=(B, S, T, u, l)$, let

$$
\mathcal{C}(\mathcal{E})=\{A \in \mathcal{A} \mid A \text { is a core outcome }\}
$$

be the core of $\mathcal{E}$.

Proposition 5.5: Consider an economy $\mathcal{E}=(B, S, T, u, l)$. Then $\mathcal{C}(\mathcal{E})=\mathcal{A}^{\mathrm{SO}}(\mathcal{E})=$ $\mathcal{A}^{\mathrm{QCCE}}(\mathcal{E}) \neq \emptyset$.

Proof: See Appendix A.5.

Q.E.D.

Proposition 5.5 shows that all main solution concepts coincide. Core outcomes correspond to QCCE outcomes, which have already been shown to correspond to stable outcomes.

Definition 5.6: An outcome $A \in \mathcal{A}$ is a strict core outcome if there is no outcome $A^{\prime} \in \mathcal{A}$ and a set of agents $N \subseteq B \cup S$ such that:

(i) For each $i \in(B \cup S) \backslash N,\left(A^{\prime}\right)^{i}=\emptyset$.

(ii) For each $j \in N, U^{j}\left(A^{\prime}\right) \geq U^{j}(A)$, with at least one strict inequality.

Analogous to the proof of Proposition 5.5, we can show that the set of strongly stable outcomes is equivalent to the set of strict core outcomes. Thus, using Example 3.6, we can also conclude that the set of strict core outcomes can be empty in the matching model with financial constraints.

\subsection{QCCE with Uniform Prices}

For markets where all trading opportunities are universally available, it is conventional to require that prices are uniform in a competitive equilibrium, i.e. each object is assigned a single price, see, e.g., Gul and Stacchetti (1999), Sun and Yang (2006), and Herings (2018).

In the absence of financial constraints, given an arbitrary competitive equilibrium with personalized prices, one can obtain both a competitive equilibrium with seller-uniform prices and a competitive equilibrium with buyer-uniform prices by adjusting the prices, while keeping the equilibrium trades the same, see, e.g., Hatfield et al. (2013), Candogan et al. (2017), and Schlegel (2019). In the presence of financial constraints, given an arbitrary 
QCCE with personalized prices, one also has to take the quantity constraints into account in order to obtain a QCCE with uniform prices. In this subsection, we present results with sufficient conditions to have QCCEs with seller-uniform prices, buyer-uniform prices, and uniform prices.

First we look at the sellers' side. Given $s \in S$, two trades $t, t^{\prime} \in T^{s}$ are said to be $s$-equivalent, denoted $t \sim^{s} t^{\prime}$, if $u^{s}(t, \cdot)=u^{s}\left(t^{\prime}, \cdot\right)$. Since $\sim^{s}$ is an equivalence relation, the collection of equivalence classes of $T^{s}$ induced by $\sim^{s}$ forms a partition of $T^{s}$, denoted by $\mathcal{T}^{s}$. A typical example of $s$-equivalent trades occurs in the assignment model with financial constraints, where the seller of an item does not care to whom the item is sold. In this case it holds that $\mathcal{T}^{s}=\left\{T^{s}\right\}$. The collection $\mathcal{T}^{S}=\cup_{s \in S} \mathcal{T}^{s}$ is a partition of $T$ with the property that for every $I \in \mathcal{T}^{S}$ there is $s \in S$ such that $I$ is a set of $s$-equivalent trades. A QCCE has seller-uniform prices if all trades that belong to the same set in $\mathcal{T}^{S}$ have the same price. The next result states the existence of a QCCE with seller-uniform prices.

Proposition 5.7: Consider an economy $\mathcal{E}=(B, S, T, u, l)$. Then there is a QCCE with seller-uniform prices.

Proof: See Appendix A.6.

Q.E.D.

The sketch of the proof of Proposition 5.7 is as follows. By Corollary 4.4, there is always a $\operatorname{QCCE}(p, q, A)$. Take some $I_{1} \in \mathcal{T}^{S}$. Then we show how to derive a QCCE $\left(p^{1}, q^{1}, A^{1}\right)$ such that $q^{1}=q, A^{1}=A$, for every $t \in T \backslash I_{1}, p_{t}^{1}=p_{t}$, and, for every $t, t^{\prime} \in I_{1}, p_{t}^{1}=p_{t^{\prime}}^{1}$. Then, by a similar argument, given $\left(p^{1}, q^{1}, A^{1}\right)$ and $I_{2} \in \mathcal{T}^{S} \backslash\left\{I_{1}\right\}$, we can derive a QCCE $\left(p^{2}, q^{2}, A^{2}\right)$ such that $q^{2}=q^{1}, A^{2}=A^{1}$, for every $t \in T \backslash I_{2}, p_{t}^{2}=p_{t}^{1}$, and, for every $t, t^{\prime} \in I_{2}$, $p_{t}^{2}=p_{t^{\prime}}^{2}$, and so on.

To define $\left(p^{1}, q^{1}, A^{1}\right)$, we adjust the prices $p$ in the following way. For each trade in $I_{1}$, we use the maximum price among all prices of trades in $I_{1}$ as the uniform price of trades in $I_{1}$. We keep the prices of other trades unchanged. More formally, we have, for each $t \in I_{1}$, $p_{t}^{1}=\max _{t^{\prime} \in I_{1}} p_{t^{\prime}}$, and for each $t \in T \backslash I_{1}, p_{t}^{1}=p_{t}$. The vector of quantity constraints $q$ and the outcome $A$ need no adjustment: $q^{1}=q$ and $A^{1}=A$. If a trade belongs to $I_{1} \cap \tau(A)$, then its price is equal to the maximum among all trades in $I_{1}$.

We now look at the buyers' side. Given $b \in B$, two trades $t, t^{\prime} \in T^{b}$ are said to be $b$-equivalent, denoted $t \sim^{b} t^{\prime}$, if $l_{t}^{b}=l_{t^{\prime}}^{b}$ and $u^{b}(t, \cdot)=u^{b}\left(t^{\prime}, \cdot\right)$. Since $\sim^{b}$ is an equivalence relation, the collection of equivalence classes of $T^{b}$ induced by $\sim^{b}$ forms a partition of $T^{b}$, denoted by $\mathcal{T}^{b}$. The collection $\mathcal{T}^{B}=\cup_{b \in B} \mathcal{T}^{b}$ is a partition of $T$ with the property that for every $I \in \mathcal{T}^{B}$ there is $b \in B$ such that $I$ is a set of $b$-equivalent trades. A QCCE has buyer-uniform prices if all trades that belong to the same set in $\mathcal{T}^{B}$ have the same price. The next result states the existence of a QCCE with buyer-uniform prices. 
Proposition 5.8: Consider an economy $\mathcal{E}=(B, S, T, u, l)$. Then there is a QCCE with buyer-uniform prices.

Proof: See Appendix A.7.

Q.E.D.

The sketch of the proof of Proposition 5.8 is as follows. By Corollary 4.4, a QCCE $(p, q, A)$ exists. Let some $I_{1} \in \mathcal{T}^{B}$ be given. Then we show how to derive a QCCE $\left(p^{1}, q^{1}, A^{1}\right)$ such that, for every $t \in T \backslash I_{1}, p_{t}^{1}=p_{t}$, and, for every $t, t^{\prime} \in I_{1}, p_{t}^{1}=p_{t^{\prime}}^{1}$. Then, by a similar argument, given $\left(p^{1}, q^{1}, A^{1}\right)$ and $I_{2} \in \mathcal{T}^{B} \backslash\left\{I_{1}\right\}$, we can derive a QCCE $\left(p^{2}, q^{2}, A^{2}\right)$ such that, for every $t \in T \backslash I_{2}, p_{t}^{2}=p_{t}^{1}$, and, for every $t, t^{\prime} \in I_{2}, p_{t}^{2}=p_{t^{\prime}}^{2}$, and so on.

The QCCE $\left(p^{1}, q^{1}, A^{1}\right)$ is derived as follows. For each trade in $I_{1}$, we use the minimum price among all prices of trades in $I_{1}$ as the uniform price of trades in $I_{1}$. The prices of other trades are kept unchanged. More precisely, we have, for each $t \in I_{1}, p_{t}^{1}=\min _{t^{\prime} \in I_{1}} p_{t^{\prime}}$ and, for each $t \in T \backslash I_{1}, p_{t}^{1}=p_{t}$. Then we adjust the vector of quantity constraints for trades in $I_{1}$. For each $t \in I_{1}$ such that $p_{t}>l_{t}^{b_{1}}$ and $p_{t}^{1}=l_{t}^{b_{1}}$ we define $q_{t}^{1}=1$. For each $t \in I_{1}$ such that $q_{t}=1$ and $p_{t}^{1}<l_{t}^{b_{1}}$, we define $q_{t}^{1}=0$. Quantity constraints for other trades remain the same. Finally, the equilibrium outcome remains the same, $A^{1}=A$.

Proposition 5.8 states that there is a QCCE with buyer-uniform prices. Using that result, it is not hard to show that there is a QCCE with buyer-uniform prices and buyeruniform quantity constraints, where the latter property means that for every $I \in \mathcal{T}^{B}$, for every $t, t^{\prime} \in I, q_{t}=q_{t^{\prime}}$.

Consider trades $t, t^{\prime}$, and $t^{\prime \prime}$ such that $t \sim^{s} t^{\prime}$ and $t \sim^{b} t^{\prime \prime}$. By Proposition 5.7 there exists a $\operatorname{QCCE}(p, q, A)$ such that $p_{t}=p_{t^{\prime}}$. By Proposition 5.8 there is a $\operatorname{QCCE}(p, q, A)$ such that $p_{t}=p_{t^{\prime \prime}}$. The next example shows that there may not be a QCCE $(p, q, A)$ such that $p_{t}=p_{t^{\prime}}=p_{t^{\prime \prime}}$

Example 5.9: Let $B=\left\{b_{1}, b_{2}\right\}$ and $S=\left\{s_{1}, s_{2}\right\}$. Let $T=\left\{t_{11}, t_{21}, t_{22}\right\}$, where $t_{i j}$ denotes the trade such that $\beta\left(t_{i j}\right)=b_{i}$ and $\sigma\left(t_{i j}\right)=s_{j}$. Let $l_{t_{11}}^{b_{1}}=4$ and $l_{t_{21}}^{b_{2}}=l_{t_{22}}^{b_{2}}=1$. Utility functions are such that, for every $i \in B \cup S, u^{i}(\emptyset)=0, u^{b_{1}}\left(t_{11}, m\right)=6-m$, $u^{b_{2}}\left(t_{21}, m\right)=u^{b_{2}}\left(t_{22}, m\right)=4-m, u^{s_{1}}\left(t_{11}, m\right)=u^{s_{1}}\left(t_{21}, m\right)=m-2$, and $u^{s_{2}}\left(t_{22}, m\right)=m$. It is easily seen that $\mathcal{T}^{B}=\left\{\left\{t_{11}\right\},\left\{t_{21}, t_{22}\right\}\right\}$ and $\mathcal{T}^{S}=\left\{\left\{t_{11}, t_{21}\right\},\left\{t_{22}\right\}\right\}$. By Proposition 5.7 there exists a $\operatorname{QCCE}(p, q, A)$ with $p_{t_{11}}=p_{t_{21}}$. It holds that $p_{t_{11}} \geq 2$, since otherwise there is no supply of $t_{11}$ by seller $s_{1}$, whereas there is demand for it by buyer $b_{1}$. By Proposition 5.8 there exists a $\operatorname{QCCE}\left(p^{\prime}, q^{\prime}, A^{\prime}\right)$ with $p_{t_{21}}^{\prime}=p_{t_{22}}^{\prime}$. It holds that $p_{t_{22}}^{\prime} \leq 1$, since otherwise there is no demand for $t_{22}$ by buyer $b_{2}$, whereas there is supply of $t_{22}$ by seller $s_{2}$. We have demonstrated that $p_{t_{21}} \neq p_{t_{21}}^{\prime}$, so there is no QCCE with prices of $t_{11}, t_{21}$, and $t_{22}$ all the same. 
In general equilibrium theory, two identical commodities are traded at the same price by the definition of a competitive equilibrium. In Example 5.9, although the trades do not involve the specification of a commodity, nothing precludes that all trades correspond to identical commodities. Example 5.9 then presents a case where no QCCE has the property that identical commodities are traded at the same price.

We now put some extra structure on the economy by making the commodity that is being traded explicit. Let $L$ be the set of commodities. The set of trades is defined as $T=B \times S \times L$. The commodity associated to a trade $t \in T$ is denoted by $\lambda(t)$, so for $t=(b, s, \ell)$ it holds that $\lambda(t)=\ell$. The concept of a commodity contains all information that is relevant for utility functions and financial constraints. In other words, the utility of a trade depends only on the traded commodity. Also, the financial constraint corresponding to a trade depends only on the traded commodity. More formally, for every $t, t^{\prime} \in T$ such that $\lambda(t)=\lambda\left(t^{\prime}\right)$ and $\beta(t)=\beta\left(t^{\prime}\right)=b$, we have that $l_{t}^{b}=l_{t^{\prime}}^{b}$ and $u^{b}(t, \cdot)=u^{b}\left(t^{\prime}, \cdot\right)$. For every $t, t^{\prime} \in T$ such that $\lambda(t)=\lambda\left(t^{\prime}\right)$ and $\sigma(t)=\sigma\left(t^{\prime}\right)=s$, we have that $u^{s}(t, \cdot)=u^{s}\left(t^{\prime}, \cdot\right)$. We refer to this set-up as an economy with commodities.

In an economy with commodities it is assumed for simplicity that any triple $(b, s, \ell) \in$ $B \times S \times L$ corresponds to a trade. This assumption is without loss of generality, since our assumptions on utility functions are sufficiently weak to allow for the case where a trade $t=(b, s, \ell)$ is such that, for every $m \in \mathbb{R}, u^{b}(t, m)<U^{b}(\emptyset)$ and, for every $m \in \mathbb{R}$, $u^{s}(t, m)<U^{s}(\emptyset)$. In other words, even under the assumption that $T=B \times S \times L$, it can be modeled that certain buyers are not interested in some commodities and certain sellers cannot supply some commodities.

A QCCE $(p, q, A)$ of an economy with commodities is said to have uniform prices if for every $t, t^{\prime} \in T$ such that $\lambda(t)=\lambda\left(t^{\prime}\right)$ we have that $p_{t}=p_{t^{\prime}}$.

In an economy with commodities, it holds that, for every $b \in B, \mathcal{T}^{b}=\{\{b\} \times S \times\{\ell\} \mid$ $\ell \in L\}$, and, for every $s \in S, \mathcal{T}^{s}=\{B \times\{s\} \times\{\ell\} \mid \ell \in L\}$. If a $\operatorname{QCCE}(p, q, A)$ of an economy with commodities has uniform prices, then it also has buyer-uniform and selleruniform prices. The next result shows that an economy with commodities has a QCCE with uniform prices.

Theorem 5.10: Consider an economy $\mathcal{E}=(B, S, T, u, l)$ with commodities. Then there is a QCCE with uniform prices.

Proof: See Appendix A.8.

Q.E.D.

The proof of Theorem 5.10 first uses the result of Proposition 5.8 to establish the existence of a $\mathrm{QCCE}(p, q, A)$ with buyer-uniform prices. This implies that $p_{t}$ can only depend on 
$\beta(t)$ and $\lambda(t)$. Next a QCCE $(\bar{p}, q, A)$ with uniform prices is constructed by defining $\bar{p}_{t^{\prime}}$ as the maximum of $p_{t}$ over all trades such that $\lambda(t)=\lambda\left(t^{\prime}\right)$.

Theorem 5.10 states that there is a QCCE with uniform prices. However, there may not exist a QCCE with uniform prices and uniform quantity constraints, where the latter property means that for every $t, t^{\prime} \in T$ such that $\lambda(t)=\lambda\left(t^{\prime}\right)$ we have that $q_{t}=q_{t^{\prime}}$. In Example 4.2, there are only two QCCEs, and both of them are QCCEs with uniform prices, i.e., $(p, q, A)$ with $p=\left(p_{t}, p_{t^{\prime}}\right)=(2,2), q=\left(q_{t}, q_{t^{\prime}}\right)=(1,0)$, and $A=\left\{\left(t^{\prime}, 2\right)\right\}$, and $\left(p^{\prime}, q^{\prime}, A^{\prime}\right)$ with $p^{\prime}=\left(p_{t}^{\prime}, p_{t^{\prime}}^{\prime}\right)=(2,2), q^{\prime}=\left(q_{t}^{\prime}, q_{t^{\prime}}^{\prime}\right)=(0,1)$, and $A^{\prime}=\{(t, 2)\}$. Nevertheless, those two QCCEs fail to have uniform quantity constraints.

Consider the assignment model with financial constraints, where the items sold by the sellers are all identical. Such an assignment model leads to an economy with commodities where the set $L$ is a singleton. Theorem 5.10 then establishes the existence of a QCCE where all trades have the same price.

\subsection{The Lattice Property}

The lattice property of competitive equilibrium payoffs and prices is intensively investigated in various directions. The lattice structure guarantees the existence of one-sided optimal outcomes and provides the possibility of designing strategy-proof mechanism, see, e.g., Shapley and Shubik (1971), Demange and Gale (1985), Hatfield and Milgrom (2005), Hatfield and Kojima (2010), Kucuksenel (2011), and Kojima et al. (2018) in matching models without financial constraints, and Ostrovsky (2008), Hatfield and Kominers (2012), Hatfield et al. (2013), Candogan et al. (2017), Fleiner et al. (2018), and Schlegel (2019) in models of trading networks.

In the presence of financial constraints, the lattice structure of prices and agents' welfare induced by QCCEs fails to hold, as exemplified next.

Example 5.11: Let $B=\left\{b_{1}, b_{2}, b_{3}, b_{4}, b_{5}\right\}$ and $S=\left\{s_{1}, s_{2}, s_{3}, s_{4}, s_{5}\right\}$. Let $t_{i j}$ be the trade such that $\beta\left(t_{i j}\right)=b_{i}$ and $\sigma\left(t_{i j}\right)=s_{j}$. Let $T=\left\{t_{11}, t_{12}, t_{21}, t_{24}, t_{32}, t_{35}, t_{43}, t_{44}, t_{53}, t_{55}\right\}$. For each $i \in B \cup S$, let $u^{i}(\emptyset)=0$. For each $b \in B$ and each $t \in T^{b}$, let $u^{b}(t, m)=u^{b}(t, 0)-m$. For each $s \in S$ and each $t \in T^{s}$, let $u^{s}(t, m)=m$. Let $u^{b_{1}}\left(t_{11}, 0\right)=u^{b_{1}}\left(t_{12}, 0\right)=10$, $u^{b_{2}}\left(t_{21}, 0\right)=10, u^{b_{2}}\left(t_{24}, 0\right)=5, u^{b_{3}}\left(t_{32}, 0\right)=10, u^{b_{3}}\left(t_{35}, 0\right)=5, u^{b_{4}}\left(t_{43}, 0\right)=2, u^{b_{4}}\left(t_{44}, 0\right)=$ $10, u^{b_{5}}\left(t_{53}, 0\right)=2$, and $u^{b_{5}}\left(t_{55}, 0\right)=10$. For each $b \in B$ and each $t \in T^{b}$, let $l_{t}^{b}=1$.

Let $(p, q, A)$ be such that

$$
\begin{aligned}
p & =\left(p_{t_{11}}, p_{t_{12}}, p_{t_{21}}, p_{t_{24}}, p_{t_{32}}, p_{t_{35}}, p_{t_{43}}, p_{t_{44}}, p_{t_{53}}, p_{t_{55}}\right)=(1,1,1,0,1,1,1,0,1,1) \\
q & =(0,0,0,0,1,0,0,0,0,1) \\
A & =\left\{\left(t_{12}, 1\right),\left(t_{21}, 1\right),\left(t_{35}, 1\right),\left(t_{44}, 0\right),\left(t_{53}, 1\right)\right\}
\end{aligned}
$$


At $(p, q, A)$, all trades corresponding to a given seller have the same price, so the seller is indifferent between supplying any of them. Every buyer gets the most preferred trade in his budget set. A quantity constraint is expected by buyers $b_{3}$ and $b_{5}$ for a trade with price equal to the corresponding financial constraint. It follows that $(p, q, A)$ is a QCCE.

Let $\left(p^{\prime}, q^{\prime}, A^{\prime}\right)$ be such that

$$
\begin{aligned}
p^{\prime} & =\left(p_{t_{11}}^{\prime}, p_{t_{12}}^{\prime}, p_{t_{21}}^{\prime}, p_{t_{24}}^{\prime}, p_{t_{32}}^{\prime}, p_{t_{35}}^{\prime}, p_{t_{43}}^{\prime}, p_{t_{44}}^{\prime}, p_{t_{53}}^{\prime}, p_{t_{55}}^{\prime}\right)=(1,1,1,1,1,0,1,1,1,0) \\
q^{\prime} & =(0,0,1,0,0,0,0,1,0,0) \\
A^{\prime} & =\left\{\left(t_{11}, 1\right),\left(t_{24}, 1\right),\left(t_{32}, 1\right),\left(t_{43}, 1\right),\left(t_{55}, 0\right)\right\}
\end{aligned}
$$

At $\left(p^{\prime}, q^{\prime}, A^{\prime}\right)$, all trades corresponding to a given seller have the same price, so the seller is indifferent between supplying any of them. Every buyer gets the most preferred trade in his budget set. A quantity constraint is expected by buyers $b_{2}$ and $b_{4}$ for a trade with price equal to the corresponding financial constraint. It follows that $\left(p^{\prime}, q^{\prime}, A^{\prime}\right)$ is a QCCE.

Let $\underline{p} \in \mathbb{R}^{T}$ be such that, for every $t \in T, p_{t}=\min \left\{p_{t}, p_{t}^{\prime}\right\}$. Then it holds that $p=(1,1,1,0,1,0,1,0,1,0)$. In the following, we show that there is no $(q, A) \in\{0,1\}^{T} \times \mathcal{A}$ such that $(p, q, A)$ is a QCCE.

By contradiction, suppose that there is $(q, A) \in\{0,1\}^{T} \times \mathcal{A}$ such that $(p, q, A)$ is a QCCE. Since $\underline{p}_{t_{35}}=\underline{p}_{t_{55}}=\underline{p}_{t_{24}}=\underline{p}_{t_{44}}=0<1=l_{t_{35}}^{b_{3}}=l_{t_{55}}^{b_{5}}=l_{t_{24}}^{b_{2}}=l_{t_{44}}^{b_{4}}$, it holds by Condition (iii) of Definition 4.1 that $q_{t_{35}}=q_{t_{55}}=q_{t_{24}}=q_{t_{44}}=0$. It follows that $\delta^{b_{4}}(\underline{p}, q)=\left\{\left\{\left(t_{44}, 0\right)\right\}\right\}$ and $\delta^{b_{5}}(\underline{p}, q)=\left\{\left\{\left(t_{55}, 0\right)\right\}\right\}$. Consequently, neither buyer $b_{4}$ nor buyer $b_{5}$ makes a transaction with seller $s_{3}$. Since $\underline{p}_{t_{43}}=\underline{p}_{t_{53}}=1$, it holds that $\delta^{s_{3}}(\underline{p}, q)=$ $\left\{\left\{\left(t_{43}, 1\right)\right\},\left\{\left(t_{53}, 1\right)\right\}\right\}$. This means that seller $s_{3}$ makes a transaction with either buyer $b_{4}$ or buyer $b_{5}$, leading to a contradiction.

We now focus on the structure of prices and agents' utilities induced by QCCEs with the same vector of quantity constraints. In such a situation, buyers form their expectations about trading opportunities in advance and then optimize their behavior.

Given prices $p, p^{\prime} \in \mathbb{R}^{T}$, we denote their component-wise minimum by $p \wedge p^{\prime}$, so, for every $t \in T,\left(p \wedge p^{\prime}\right)_{t}=\min \left\{p_{t}, p_{t}^{\prime}\right\}$. The component-wise maximum is denoted by $p \vee p^{\prime}$, so, for every $t \in T,\left(p \vee p^{\prime}\right)_{t}=\max \left\{p_{t}, p_{t}^{\prime}\right\}$. For two outcomes $A, A^{\prime} \in \mathcal{A}$, we define $A \wedge A^{\prime}$ and $A \vee A^{\prime}$ by

$$
\begin{aligned}
& A \wedge A^{\prime}=\left(\cup_{b \in B}\left\{A^{b} \mid U^{b}(A)>U^{b}\left(A^{\prime}\right)\right\}\right) \cup\left(\cup_{b \in B}\left\{\left(A^{\prime}\right)^{b} \mid U^{b}(A) \leq U^{b}\left(A^{\prime}\right)\right\}\right), \\
& A \vee A^{\prime}=\left(\cup_{b \in B}\left\{\left(A^{\prime}\right)^{b} \mid U^{b}(A)>U^{b}\left(A^{\prime}\right)\right\}\right) \cup\left(\cup_{b \in B}\left\{A^{b} \mid U^{b}(A) \leq U^{b}\left(A^{\prime}\right)\right\}\right) .
\end{aligned}
$$

Theorem 5.12: Consider an economy $\mathcal{E}=(B, S, T, u, l)$. Let $(p, q, A)$ and $\left(p^{\prime}, q, A^{\prime}\right)$ be $Q C C E s$ for $\mathcal{E}$. Then $\left(p \wedge p^{\prime}, q, A \wedge A^{\prime}\right)$ and $\left(p \vee p^{\prime}, q, A \vee A^{\prime}\right)$ are $Q C C E s$ for $\mathcal{E}$ as well. 
Theorem 5.12 shows two ways to derive a QCCE from two arbitrary QCCEs $(p, q, A)$ and $\left(p^{\prime}, q, A^{\prime}\right)$ with the same vector of quantity constraints. In the first way we obtain the QCCE $\left(p \wedge p^{\prime}, q, A \wedge A^{\prime}\right)$ by setting the price of each trade as the component-wise minimum price between the two QCCEs and assigning to each buyer the trade with the maximum utility. In the second way, we generate the QCCE $\left(p \vee p^{\prime}, q, A \vee A^{\prime}\right)$ by setting the price of each trade as the component-wise maximum price between the two QCCEs and assigning to each buyer the trade with the minimum utility.

Given an economy $\mathcal{E}=(B, S, T, u, l)$ and a vector of quantity constraints $q \in\{0,1\}^{T}$, let

$$
P(\mathcal{E}, q)=\left\{p \in \mathbb{R}^{T} \mid \exists A \in \mathcal{A} \text { such that }(p, q, A) \text { is a QCCE of } \mathcal{E}\right\}
$$

be the set of QCCE prices for a given $q$, and let

$$
\begin{aligned}
& V^{B}(\mathcal{E}, q)=\left\{\left(V^{b}\right)_{b \in B} \in \mathbb{R}^{B} \mid \exists(p, A) \in \mathbb{R}^{T} \times \mathcal{A} \text { s.t. }(p, q, A) \text { is a QCCE of } \mathcal{E} \text { and } V^{b}=U^{b}(A)\right\} \\
& V^{S}(\mathcal{E}, q)=\left\{\left(V^{s}\right)_{s \in S} \in \mathbb{R}^{S} \mid \exists(p, A) \in \mathbb{R}^{T} \times \mathcal{A} \text { s.t. }(p, q, A) \text { is a QCCE of } \mathcal{E} \text { and } V^{s}=U^{s}(A)\right\}
\end{aligned}
$$

be the set of utilities generated by the QCCEs for a given $q$.

Theorem 5.12 implies that $P(\mathcal{E}, q), V^{B}(\mathcal{E}, q)$, and $V^{S}(\mathcal{E}, q)$ are all lattices. ${ }^{3}$ However, $P(\mathcal{E}, q), V^{B}(\mathcal{E}, q)$, and $V^{S}(\mathcal{E}, q)$ may not be complete lattices. The reason that these lattices may not be complete is not only caused by the possibility of unbounded prices as allowed for by assumptions (b-i) and (s-i) of the utility functions. Indeed, in the absence of financial constraints, if for each $i \in B \cup S$ and each $t \in T^{i}$, the range of $u^{i}(t, \cdot)$ is all of $\mathbb{R}$, then $P(\mathcal{E}, q), V^{B}(\mathcal{E}, q)$, and $V^{S}(\mathcal{E}, q)$ are all complete lattices (Demange and Gale, 1985). However, even if we impose this assumption in the presence of financial constraints, the lattices $P(\mathcal{E}, q), V^{B}(\mathcal{E}, q)$, and $V^{S}(\mathcal{E}, q)$ may not be complete, as exemplified below.

Example 5.13: Consider the economy as defined in Example 5.3. Let $q=(0,0)$. Then

$$
\begin{aligned}
P(\mathcal{E}, q) & =\left\{\left(p_{t}, p_{t^{\prime}}\right) \in \mathbb{R}^{2} \mid p_{t}, p_{t^{\prime}} \in(2,3] \text { and } p_{t} \leq p_{t^{\prime}}\right\}, \\
V^{B}(\mathcal{E}, q) & =\left\{\left(V^{b_{1}}, V^{b_{2}}\right) \in \mathbb{R}^{2} \mid V^{b_{1}}=0 \text { and } 1 \leq V^{b_{2}}<2\right\}, \\
V^{S}(\mathcal{E}, q) & =\left\{V^{s_{1}} \in \mathbb{R} \mid 2<V^{s_{1}} \leq 3\right\} .
\end{aligned}
$$

The lattices $P(\mathcal{E}, q), V^{B}(\mathcal{E}, q)$, and $V^{S}(\mathcal{E}, q)$ are clearly not complete.

\footnotetext{
${ }^{3}$ The definitions of lattice and complete lattice correspond to the standard ones for matching models, see, e.g., Kucuksenel (2011).
} 
If for each $t \in T, q_{t}=0$, then a QCCE is a competitive equilibrium. Thus, the lattice structure of competitive equilibrium prices and agents' utilities in the presence of financial constraints can be also derived from Theorem 5.12.

Corollary 5.14: Consider an economy $\mathcal{E}=(B, S, T, u, l)$. Let $q=(0, \ldots, 0)$. Then $P(\mathcal{E}, q), V^{B}(\mathcal{E}, q)$, and $V^{S}(\mathcal{E}, q)$ are all lattices.

Theorem 5.12 and Corollary 5.14 involve equilibria with personalized prices. It is immediate that the same results apply when prices are required to be buyer-uniform or seller-uniform. For economies with commodities, the same results apply for the case of uniform prices.

In the assignment model with financial constraints described by Example 2.2, van der Laan et al. (2018) show that the set of prices at which there is no overdemand forms a lower-semi lattice. In such a model, Corollary 5.14 implies that the set of competitive equilibrium prices is a lattice. The set of competitive equilibrium prices is strictly included in the set of prices with no overdemand, so our result complements the one in van der Laan et al. (2018).

\subsection{Rural Hospital Theorem}

Roth (1986) considers a many-to-one matching model and shows that all stable matchings have the same set of matched doctors and hospitals. This invariance property of stable matchings is known as the rural hospital theorem. The rural hospital theorem has been extended in various directions, like matching models without constraints, e.g., Demange and Gale (1985), Hatfield and Milgrom (2005), Hatfield and Kojima (2010), matching models with constraints, e.g., Kojima et al. (2018), and models of trading networks, e.g., Hatfield and Kominers (2012), Fleiner et al. (2018), and Schlegel (2019).

The next example shows that the rural hospital theorem fails in the presence of financial constraints.

Example 5.15: Let $T=\left\{t_{11}, t_{12}, t_{21}, t_{22}\right\}$, where $t_{i j}$ denotes the trade such that $\beta\left(t_{i j}\right)=b_{i}$ and $\sigma\left(t_{i j}\right)=s_{j}$. Let $l_{t_{11}}^{b_{1}}=l_{t_{12}}^{b_{1}}=1$ and $l_{t_{21}}^{b_{2}}=l_{t_{22}}^{b_{2}}=2$. Utility functions are such that, for every $i \in B \cup S, u^{i}(\emptyset)=0, u^{b_{1}}\left(t_{11}, m\right)=6-m, u^{b_{1}}\left(t_{12}, m\right)=1-m, u^{b_{2}}\left(t_{21}, m\right)=2.5-m$, $u^{b_{2}}\left(t_{22}, m\right)=4-m, u^{s_{1}}\left(t_{11}, m\right)=u^{s_{1}}\left(t_{21}, m\right)=m-1, u^{s_{2}}\left(t_{12}, m\right)=m-1$, and $u^{s_{2}}\left(t_{22}, m\right)=$ $m$. Let $p=\left(p_{t_{11}}, p_{t_{12}}, p_{t_{21}}, p_{t_{22}}\right)=(1,1,1,2)$ and $q=\left(q_{t_{11}}, q_{t_{12}}, q_{t_{21}}, q_{t_{22}}\right)=(1,0,0,0)$. Then $A=\left\{\left(t_{22}, 2\right)\right\}$ is the only outcome such that $(p, q, A)$ is a QCCE.

Let $p^{\prime}=\left(p_{t_{11}}^{\prime}, p_{t_{12}}^{\prime}, p_{t_{21}}^{\prime}, p_{t_{22}}^{\prime}\right)=(1,1.5,0.5,1.5)$ and $q^{\prime}=\left(q_{t_{11}}^{\prime}, q_{t_{12}}^{\prime}, q_{t_{21}}^{\prime}, q_{t_{22}}^{\prime}\right)=(0,1,0,0)$.

For outcome $A^{\prime}=\left\{\left(t_{11}, 1\right),\left(t_{22}, 1.5\right)\right\}$, it holds that $\left(p^{\prime}, q^{\prime}, A^{\prime}\right)$ is a QCCE. Next, we show that there is no outcome $A^{\prime \prime}$ such that $\left(p^{\prime}, q^{\prime}, A^{\prime \prime}\right)$ is a QCCE and $\tau\left(A^{\prime \prime}\right)=\left\{t_{22}\right\}$, 
which means a failure of the rural hospital theorem. Suppose $A^{\prime \prime}$ is such that $\left(p^{\prime}, q^{\prime}, A^{\prime \prime}\right)$ is a QCCE and $\tau\left(A^{\prime \prime}\right)=\left\{t_{22}\right\}$. It follows that $\left(A^{\prime \prime}\right)^{b_{1}}=\emptyset$, so $\emptyset \in \delta^{b_{1}}\left(p^{\prime}, q^{\prime}\right)$. Since $p_{t_{11}}^{\prime}=1$ and $q_{t_{11}}^{\prime}=0$, it holds that $\delta^{b_{1}}\left(p^{\prime}, q^{\prime}\right)=\left\{\left\{\left(t_{11}, 1\right)\right\}\right\}$ and we have obtained a contradiction.

We now establish a version of the rural hospital theorem that holds for QCCEs with a given vector of quantity constraints.

Theorem 5.16: Consider an economy $\mathcal{E}=(B, S, T, u, l)$. Let $(p, q, A)$ be a $Q C C E$ for $\mathcal{E}$ and $p^{\prime} \in P(\mathcal{E}, q)$. Then there is an outcome $A^{\prime} \in \mathcal{A}$ with $\beta\left(\tau\left(A^{\prime}\right)\right)=\beta(\tau(A))$ and $\sigma\left(\tau\left(A^{\prime}\right)\right)=\sigma(\tau(A))$ such that $\left(p^{\prime}, q, A^{\prime}\right)$ is a $Q C C E$ for $\mathcal{E}$.

Proof: See Appendix A.10.

Q.E.D.

Theorem 5.16 considers two arbitrary QCCEs with the same vector of quantity constraints. It shows that there is a QCCE with the same set of agents signing contracts as in the first QCCE and prices as given in the second QCCE. To prove Theorem 5.16, we first use the $\mathrm{QCCE}(p, q, A)$ and prices $p^{\prime}$ to construct a QCCE $\left(p \vee p^{\prime}, q, A^{\prime \prime}\right)$ such that the sets of agents choosing the no-trade option are the same at $(p, q, A)$ and $\left(p \vee p^{\prime}, q, A^{\prime \prime}\right)$, i.e., $B \backslash \beta(\tau(A))=B \backslash \beta\left(\tau\left(A^{\prime \prime}\right)\right)$ and $S \backslash \sigma(\tau(A))=S \backslash \sigma\left(\tau\left(A^{\prime \prime}\right)\right)$. Then we use the QCCE $\left(p \vee p^{\prime}, q, A^{\prime \prime}\right)$ and prices $p^{\prime}$ to construct another QCCE $\left(\left(p \vee p^{\prime}\right) \wedge p^{\prime}, q, A^{\prime}\right)$ such that the sets of agents choosing the no-trade option are the same at those two QCCEs, i.e., $B \backslash \beta\left(\tau\left(A^{\prime \prime}\right)\right)=B \backslash \beta\left(\tau\left(A^{\prime}\right)\right)$ and $S \backslash \sigma\left(\tau\left(A^{\prime \prime}\right)\right)=S \backslash \sigma\left(\tau\left(A^{\prime}\right)\right)$. So we have $B \backslash \beta(\tau(A))=$ $B \backslash \beta\left(\tau\left(A^{\prime}\right)\right)$ and $S \backslash \sigma(\tau(A))=S \backslash \sigma\left(\tau\left(A^{\prime}\right)\right)$. The result now follows from observing that $\left(p \vee p^{\prime}\right) \wedge p^{\prime}=p^{\prime}$.

If for each $t \in T, q_{t}=0$, then a QCCE is a competitive equilibrium. Thus, the rural hospital theorem of competitive equilibria can also be derived from Theorem 5.16. Demange and Gale (1985) establish the rural hospital theorem of stable outcomes in the matching model without financial constraints as described by Example 2.1. Notice that by Theorem 5.1, their result is also a by-product of Theorem 5.16.

It is immediate that the appropriate analogues of Theorem 5.16 apply to QCCEs with buyer-uniform prices, seller-uniform prices, and uniform prices.

\section{Financial Constraints versus Price Controls}

Matching markets with price controls are such that prices are restricted to be in between price floors and price ceilings, e.g., Talman and Yang (2008), Andersson and Svensson (2014), and Herings (2018). An important difference between price controls and financial constraints is that price controls are generally publicly known, whereas financial constraints 
are often private information. As a consequence of this difference, there is a constrained efficient and strategy-proof mechanism in the assignment model with price controls, see, e.g., Andersson and Svensson (2014), whereas in the assignment model with financial constraints such a mechanism does not exist, see, e.g., Dobzinski et al. (2012).

In the following, we elaborate on the connection between matching markets with financial constraints and those with price controls. We first introduce a special case of the model with price controls as introduced in Herings (2018), where only price ceilings are allowed. For each $t \in T$, let $\bar{p}_{t} \in \overline{\mathbb{R}}_{+}$be a price ceiling for the price of trade $t$. Let $\bar{p}=\left(\bar{p}_{t}\right)_{t \in T} \in \overline{\mathbb{R}}_{+}^{T}$ be a vector of price ceilings. A matching model with price controls is described by $\overline{\mathcal{E}}=(B, S, T, u, \bar{p})$.

The set of feasible contracts is given by $\bar{Y}^{\mathrm{p}}=\left\{(t, m) \in T \times \mathbb{R} \mid m \leq \bar{p}_{t}\right\}$. Let $\overline{\mathcal{A}}^{\mathrm{p}}$ be the collection of outcomes resulting from $\bar{Y}^{\mathrm{p}}$.

The budget set and the demand set of buyer $b \in B$ at $(p, q) \in \mathbb{R}^{T} \times\{0,1\}^{T}$ are given by

$$
\begin{aligned}
& \bar{\gamma}^{b}(p, q)=\left\{\{c\} \in \overline{\mathcal{A}}^{\mathrm{p}} \mid \tau(c) \in T^{b}, \mu(c)=p_{\tau(c)}, \text { and } q_{\tau(c)}=0\right\} \cup\{\emptyset\} . \\
& \bar{\delta}^{b}(p, q)=\underset{A^{b} \in \bar{\gamma}^{b}(p, q)}{\arg \max } U^{b}\left(A^{b}\right) .
\end{aligned}
$$

The budget set and the demand set of seller $s \in S$ at $p \in \mathbb{R}^{T}$ are given by

$$
\begin{aligned}
& \bar{\gamma}^{s}(p)=\left\{\{c\} \in \overline{\mathcal{A}}^{\mathrm{p}} \mid \tau(c) \in T^{s} \text { and } \mu(c)=p_{\tau(c)}\right\} \cup\{\emptyset\} . \\
& \bar{\delta}^{s}(p)=\underset{A^{s} \in \bar{\gamma}^{s}(p)}{\arg \max } U^{s}\left(A^{s}\right) .
\end{aligned}
$$

Definition 6.1 (Herings, 2018): An element $(p, q, A) \in \mathbb{R}^{T} \times\{0,1\}^{T} \times \overline{\mathcal{A}}^{\mathrm{p}}$ is a Drèze equilibrium $(D E)$ for the economy $\overline{\mathcal{E}}=(B, S, T, u, \bar{p})$ if:

(i) For each $b \in B, A^{b} \in \bar{\delta}^{b}(p, q)$.

(ii) For each $s \in S, A^{s} \in \bar{\delta}^{s}(p)$.

(iii) For each $t \in T, p_{t} \leq \bar{p}_{t}$.

(iv) For each $t \in T$, if $p_{t}<\bar{p}_{t}$, then $q_{t}=0$.

We now convert an economy $\mathcal{E}=(B, S, T, u, l)$ with financial constraints into an economy $\overline{\mathcal{E}}=(B, S, T, u, \bar{p})$ with price ceilings by setting, for every $t \in T, \bar{p}_{t}=l_{t}^{\beta(t)}$. We call $\overline{\mathcal{E}}$ the economy with price ceilings associated to $\mathcal{E}$. Since $\bar{Y}=\left\{(t, m) \in T \times \mathbb{R} \mid m \leq l_{t}^{\beta(t)}\right\}$ and $\bar{Y}^{\mathrm{p}}=\left\{(t, m) \in T \times \mathbb{R} \mid m \leq \bar{p}_{t}\right\}$, it holds that $\bar{Y}=\bar{Y}^{\mathrm{p}}$. It follows that $\mathcal{A}=\overline{\mathcal{A}}^{\mathrm{p}}$ and $\mathcal{A}^{\mathrm{SO}}(\mathcal{E})=\mathcal{A}^{\mathrm{SO}}(\overline{\mathcal{E}})$. Let

$$
\overline{\mathcal{A}}^{\mathrm{DE}}(\overline{\mathcal{E}})=\left\{A \in \overline{\mathcal{A}}^{\mathrm{p}} \mid \text { there is }(p, q) \in \mathbb{R}^{T} \times\{0,1\}^{T} \text { such that }(p, q, A) \text { is a DE }\right\} .
$$


Corollary 6.4 in Herings (2018) states the equivalence between $\overline{\mathcal{A}}^{\mathrm{SO}}(\overline{\mathcal{E}})$ and $\overline{\mathcal{A}}^{\mathrm{DE}}(\overline{\mathcal{E}})$. Combining this with Theorem 5.1, we obtain the following corollary.

Corollary 6.2: Consider an economy $\mathcal{E}=(B, S, T, u, l)$ and let $\overline{\mathcal{E}}$ be the associated economy with price ceilings. It holds that $\mathcal{A}^{\mathrm{QCCE}}(\mathcal{E})=\mathcal{A}^{\mathrm{SO}}(\mathcal{E})=\mathcal{A}^{\mathrm{SO}}(\overline{\mathcal{E}})=\mathcal{A}^{\mathrm{DE}}(\overline{\mathcal{E}}) \neq \emptyset$.

However, a QCCE of $\mathcal{E}$ is not necessarily a DE of the associated economy with price ceilings $\overline{\mathcal{E}}$, because the price of a trade $t$ in a $\operatorname{QCCE}(p, q, A)$ can exceed buyer $\beta(t)$ 's financial constraint on $t$, i.e., $p_{t}>l_{t}^{\beta(t)}=\bar{p}_{t}$, which violates Condition (iii) of Definition 6.1.

A vector of quantity constraints that is compatible with a QCCE may not be compatible with a DE as is demonstrated in the next example. In this example, the QCCE is such that there are no quantity constraints, so it is a competitive equilibrium.

Example 6.3: We take the same primitives as in Example 5.3. Let price ceilings be given by $\bar{p}_{t}=l_{t}^{b_{1}}=2$ and $\bar{p}_{t^{\prime}}=l_{t^{\prime}}^{b_{2}}=3$. It is easily verified that $(p, q, A)$ with $p=\left(p_{t}, p_{t^{\prime}}\right)=(3,3)$, $q=\left(q_{t}, q_{t^{\prime}}\right)=(0,0)$, and $A=\left\{\left(t^{\prime}, 3\right)\right\}$ is a QCCE. In fact, it is a competitive equilibrium with uniform prices. It is not a DE since $p_{t}=3>2=\bar{p}_{t}$. In fact, it is easily seen that there is no DE with $q=(0,0)$. At any prices $\left(p_{t}, p_{t^{\prime}}\right) \leq(2,3)=\left(\bar{p}_{t}, \bar{p}_{t^{\prime}}\right)$, it holds that $\bar{\delta}^{b_{1}}(p,(0,0))=\left\{\left\{\left(t, p_{t}\right)\right\}\right\}$ and $\bar{\delta}^{b_{2}}(p,(0,0))=\left\{\left\{\left(t^{\prime}, p_{t^{\prime}}\right)\right\}\right\}$. Since the seller can supply at most one unit, a DE with $q=(0,0)$ does not exist.

The next result states that DEs are a refinement of QCCEs.

Theorem 6.4: Consider an economy $\mathcal{E}=(B, S, T, u, l)$ and let $\overline{\mathcal{E}}$ be the associated economy with price ceilings. If $(p, q, A)$ is a DE for $\overline{\mathcal{E}}$, then $(p, q, A)$ is a $Q C C E$ for $\mathcal{E}$.

Proof: See Appendix A.11.

Q.E.D.

If we fix a vector of quantity constraints and assume that the agents' utility functions satisfy the finiteness assumption imposed by Demange and Gale (1985), see Example 2.1, then the sets of prices and utilities at DEs with that vector of quantity constraints are complete lattices. As shown in Example 5.13, this is not the case for QCCEs.

Similar to matching markets with financial constraints, the rural hospital theorem fails to hold for matching markets with price controls. But for DEs with the same vector of quantity constraints, a result parallel to Theorem 5.16 can still be established. 


\section{Concluding Remarks}

We show how to conduct the competitive analysis in the one-to-one matching with contracts model when there are financial constraints. In such a model, although a stable outcome always exists, there is in general no competitive equilibrium as defined in the standard way. We argue that in some cases buyers should be allowed to hold expectations that there is no supply of certain trades. This may occur exactly when the price of the trade is equal to the financial constraint or above. At our equilibrium notion, called quantity-constrained competitive equilibium (QCCE), such expectations are confirmed and thereby rational. We show that a QCCE always exists.

We analyze the properties of QCCEs from a number of perspectives. First, we establish the equivalence between the set of stable outcomes and the set of QCCE outcomes. Second, we show the equivalence between the core and the set of QCCE outcomes. Third, we present sufficient conditions to guarantee the existence of QCCEs with buyer-uniform prices, selleruniform prices, and uniform prices. Fourth, we show that given a fixed vector of quantity constraints, the sets of prices and agents' utilities induced by QCCEs with that vector of quantity constraints are lattices, though not necessarily complete ones. Fifth, we show that given a fixed vector of quantity constraints, the rural hospital theorem holds for QCCEs with that vector of quantity constraints.

We also discuss the relation between economies with financial constraints and those with price controls. We associate to each economy with financial constraints a corresponding economy with price controls. The standard equilibrium notion for the economy with price controls is the Drèze equilibrium (DE). We show that the set of QCCE outcomes of an economy is equivalent to the set of $\mathrm{DE}$ outcomes of the associated economy with price controls. However, QCCE prices and quantity constraints may not be DE prices and quantity constraints. The set of DEs is a subset of the set of QCCEs, where the inclusion can be strict.

Throughout the paper, we focus on the situation where only buyers face financial constraints. The equilibrium concept of QCCE and the results of this paper can also be extended to the case where sellers face financial constraints or both buyers and sellers face financial constraints.

\section{Appendix}

\section{A.1 Proof of Lemma 3.3}

We prove Lemma 3.3 in four steps.

Step 1: There is $K \geq 0$ such that $A^{K+1}=Z^{K}$, i.e., the adjustment process terminates in 
a finite number of steps.

Suppose not. Then, for every $k \geq 0$, it holds that $A^{k+1} \subsetneq Z^{k}$ and therefore $Y^{k+1} \subsetneq Y^{k}$. Since $Y^{0}=\bar{Y}^{\varepsilon}$ and $\bar{Y}^{\varepsilon}$ is finite, this leads to a contradiction.

Step 2: $A^{K+1}$ is an outcome.

For each $i \in \beta\left(\tau\left(A^{K+1}\right)\right) \cup \sigma\left(\tau\left(A^{K+1}\right)\right)$, it holds that $\left|\left(A^{K+1}\right)^{i}\right|=1$. For each $i \in$ $(B \cup S) \backslash\left(\beta\left(\tau\left(A^{K+1}\right)\right) \cup \sigma\left(\tau\left(A^{K+1}\right)\right)\right)$, it holds that $\left|\left(A^{K+1}\right)^{i}\right|=0$.

Step 3: Condition (i) of Definition 3.1 for $\bar{Y}^{\varepsilon}$ holds at $A^{K+1}$, i.e., for each $i \in B \cup S$, $\left(A^{K+1}\right)^{i} \in \mathrm{Ch}^{i}\left(A^{K+1}\right)$.

For each $i \in(B \cup S) \backslash\left(\beta\left(A^{K+1}\right) \cup \sigma\left(A^{K+1}\right)\right)$, since $\left|\left(A^{K+1}\right)^{i}\right|=0$ it holds that $\left(A^{K+1}\right)^{i}=$ $\emptyset \in \mathrm{Ch}^{i}\left(A^{K+1}\right)$. For each $b \in \beta\left(A^{K+1}\right)$, since buyer $b$ has chosen $\left(A^{K+1}\right)^{b}$, it holds that $U^{b}\left(A^{K+1}\right) \geq U^{b}(\emptyset)$, so $\left(A^{K+1}\right)^{b} \in \mathrm{Ch}^{b}\left(A^{K+1}\right)$. For each $s \in \sigma\left(A^{K+1}\right)$, since seller $s$ accepts $\left(A^{K+1}\right)^{s}$, it holds that $U^{s}\left(A^{K+1}\right) \geq U^{s}(\emptyset)$, so $\left(A^{K+1}\right)^{s} \in \mathrm{Ch}^{s}\left(A^{K+1}\right)$.

Step 4: Condition (ii) of Definition 3.1 for $\bar{Y}^{\varepsilon}$ holds at $A^{K+1}$, i.e., there is no $c=(t, m) \in$ $\bar{Y}^{\varepsilon}$ such that $U^{\beta(t)}(\{c\})>U^{\beta(t)}\left(A^{K+1}\right)$ and $U^{\sigma(t)}(\{c\})>U^{\sigma(t)}\left(A^{K+1}\right)$.

By contradiction, suppose that there is $c=(t, m) \in \bar{Y}^{\varepsilon}$ such that $U^{\beta(t)}(\{c\})>$ $U^{\beta(t)}\left(A^{K+1}\right)$ and $U^{\sigma(t)}(\{c\})>U^{\sigma(t)}\left(A^{K+1}\right)$. Since $U^{\beta(t)}(\{c\})>U^{\beta(t)}\left(A^{K+1}\right)$, there is $k<K$ such that $\beta(t)$ chooses $\{c\}$, but $\{c\}$ is rejected by $\sigma(t)$. This implies $U^{\sigma(t)}\left(A^{k+1}\right) \geq$ $U^{\sigma(t)}(\{c\})$. Since the welfare of seller $\sigma(t)$ is non-decreasing, it follows that $U^{\sigma(t)}\left(A^{K+1}\right) \geq$ $U^{\sigma(t)}(\{c\})$, contradicting $U^{\sigma(t)}(\{c\})>U^{\sigma(t)}\left(A^{K+1}\right)$.

Q.E.D.

\section{A.2 Proof of Proposition 3.4}

Take some $\varepsilon>0$ and let $\left(\varepsilon_{n}\right)_{n \in \mathbb{Z}^{+}}$be the sequence such that $\varepsilon_{n}=\varepsilon / 2^{n}$. It holds that $\varepsilon_{n} \rightarrow 0$ as $n \rightarrow+\infty$. By Lemma 3.3, for each $\varepsilon_{n}>0$, there is a stable outcome $A^{\varepsilon_{n}}$ for $\bar{Y}^{\varepsilon_{n}}$. Since the set of trades is finite, there is a subsequence $\left(\varepsilon_{n}^{\prime}\right)_{n \in \mathbb{Z}^{+}}$of $\left(\varepsilon_{n}\right)_{n \in \mathbb{Z}^{+}}$such that the set of trades $\tau\left(A^{\varepsilon_{n}^{\prime}}\right)$ does not depend on $\varepsilon_{n}^{\prime}$. We denote this set of trades by $T^{\prime}$. Consider the following two cases.

Case 1: $T^{\prime}=\emptyset$.

We show that $A=\emptyset$ is a stable outcome for $\bar{Y}$.

For each $i \in B \cup S$, it holds that $A^{i}=\emptyset$. Thus $A$ is an outcome. For each $i \in B \cup S$, it holds that $A^{i}=\emptyset \in \mathrm{Ch}^{i}(\emptyset)$, so Condition (i) of Definition 3.1 holds.

To show Condition (ii) of Definition 3.1 holds, by contradiction, suppose that there is $c=(t, m) \in \bar{Y}$ such that $U^{\beta(t)}(\{c\})>U^{\beta(t)}(\emptyset)$ and $U^{\sigma(t)}(\{c\})>U^{\sigma(t)}(\emptyset)$. For sufficiently small $\varepsilon_{n}^{\prime}$, there is $c^{\prime}=\left(t, m^{\prime}\right) \in \bar{Y}^{\varepsilon_{n}^{\prime}}$ such that $U^{\beta(t)}\left(\left\{c^{\prime}\right\}\right)>U^{\beta(t)}(\emptyset)$ and $U^{\sigma(t)}\left(\left\{c^{\prime}\right\}\right)>$ $U^{\sigma(t)}(\emptyset)$. Thus $U^{\beta(t)}\left(\left\{c^{\prime}\right\}\right)>U^{\beta(t)}(\emptyset)=U^{\beta(t)}\left(A^{\varepsilon_{n}^{\prime}}\right)$ and $U^{\sigma(t)}\left(\left\{c^{\prime}\right\}\right)>U^{\sigma(t)}(\emptyset)=U^{\sigma(t)}\left(A^{\varepsilon_{n}^{\prime}}\right)$, contradicting that $A^{\varepsilon_{n}^{\prime}}$ is a stable outcome for $\bar{Y}^{\varepsilon_{n}^{\prime}}$.

Case 2: $T^{\prime} \neq \emptyset$.

Let a trade $t \in T^{\prime}$ be given. Let $b=\beta(t)$ and $s=\sigma(t)$. The bounds $\underline{m}_{t}^{s}$ and $\bar{m}_{t}^{b}$ are such 
that, for every $n \in \mathbb{Z}^{+}$, for $c_{n} \in A^{\varepsilon_{n}^{\prime}}$ with $\tau\left(c_{n}\right)=t$ it holds that $\underline{m}_{t}^{s} \leq \mu\left(c_{n}\right) \leq \bar{m}_{t}^{b}$. Since the set $T^{\prime}$ is finite and monetary payments are uniformly bounded, the sequence $\left(A^{\varepsilon_{n}^{\prime}}\right)_{n \in \mathbb{Z}^{+}}$ has a convergent subsequence $\left(A_{n}^{\varepsilon_{n}^{\prime \prime}}\right)_{n \in \mathbb{Z}^{+}}$with limit $A$.

For every $(t, m) \in A$ it holds that $\underline{m}_{t}^{\sigma(t)} \leq m \leq \bar{m}_{t}^{\beta(t)}$.

We show that $A$ is a stable outcome for $\bar{Y}$.

Since, for every $n \in \mathbb{Z}^{+}, A^{\varepsilon_{n}^{\prime \prime}}$ is an outcome, it follows that $A$ is an outcome. It is straightforward that for each $i \in B \cup S$ such that $A^{i}=\emptyset, A^{i} \in \mathrm{Ch}^{i}(A)$. In the following, we show that for each $i \in B \cup S$ such that $A^{i} \neq \emptyset, A^{i} \in \mathrm{Ch}^{i}(A)$. By contradiction, suppose that there is $i \in B \cup S$ such that $A^{i} \neq \emptyset$ and $A^{i} \notin \mathrm{Ch}^{i}(A)$. Then $U^{i}(\emptyset)>U^{i}(A)$. Then, by selecting a sufficiently large $n$, it holds that $U^{i}(\emptyset)>U^{i}\left(A^{\varepsilon_{n}^{\prime \prime}}\right)$, contradicting that $A^{\varepsilon_{n}^{\prime \prime}}$ is a stable outcome for $\bar{Y}^{\varepsilon_{n}^{\prime \prime}}$. Thus, Condition (i) of Definition 3.1 holds.

To show that Condition (ii) of Definition 3.1 holds, by contradiction, suppose that there is $c=(t, m) \in \bar{Y}$ such that $U^{\beta(t)}(\{c\})>U^{\beta(t)}(A)$ and $U^{\sigma(t)}(\{c\})>U^{\sigma(t)}(A)$. For $n$ sufficiently large, there is $c^{\prime}=\left(t, m^{\prime}\right) \in \bar{Y}_{n}^{\varepsilon_{n}^{\prime \prime}}$ such that $U^{\beta(t)}\left(\left\{c^{\prime}\right\}\right)>U^{\beta(t)}\left(A_{n}^{\varepsilon_{n}^{\prime \prime}}\right)$ and $U^{\sigma(t)}\left(\left\{c^{\prime}\right\}\right)>U^{\sigma(t)}\left(A^{\varepsilon_{n}^{\prime \prime}}\right)$, contradicting that $A^{\varepsilon_{n}^{\prime \prime}}$ is a stable outcome for $\bar{Y}^{\varepsilon_{n}^{\prime \prime}}$.

Consequently, $A$ is a stable outcome for $\bar{Y}$.

Q.E.D.

\section{A.3 Proof of Proposition 4.3}

For every $b \in B$, for every $t \in T^{b}$, recall that $m_{t}^{b} \in \mathbb{R}$ satisfies $u^{b}\left(t, m_{t}^{b}\right) \leq u^{b}(\emptyset)$. Moreover, we have $\bar{m}_{t}^{b}=\min \left\{l_{t}^{b}, m_{t}^{b}\right\}$. For every $b \in B$, for every $t \in T^{b}$, one of the following three cases holds:

$(b-1)$ For every $m \in \mathbb{R}, U^{b}(A)>u^{b}(t, m)$.

$(b-2)$ For some $m^{\prime} \leq \bar{m}_{t}^{b}, U^{b}(A)=u^{b}\left(t, m^{\prime}\right)$.

$(b-3) l_{t}^{b}<m_{t}^{b}$ and $U^{b}(A)<u^{b}\left(t, l_{t}^{b}\right)$.

For every $s \in S$, for every $t \in T^{s}$, recall that $\underline{m}_{t}^{s} \in \mathbb{R}$ satisfies $u^{s}\left(t, \underline{m}_{t}^{s}\right) \leq u^{s}(\emptyset)$. Since $A$ is a stable outcome it follows by Condition (i) of Definition 3.1 that $U^{s}(A) \geq u^{s}(\emptyset) \geq$ $u^{s}\left(t, \underline{m}_{t}^{s}\right)$.

For every $s \in S$, for every $t \in T^{s}$, one of the following two cases holds:

$(s-1)$ For every $m \in \mathbb{R}, U^{s}(A)>u^{s}(t, m)$.

$(s-2)$ For some $m^{\prime \prime} \geq \underline{m}_{t}^{s}, U^{s}(A)=u^{s}\left(t, m^{\prime \prime}\right)$.

Step 1: Let $t \in T, b=\beta(t)$, and $s=\sigma(t)$. We first show statements (i) and (ii): (i) If $l_{t}^{b}<m_{t}^{b}, U^{b}(A)<u^{b}\left(t, l_{t}^{b}\right)$, and there is some $m^{\prime \prime} \geq \underline{m}_{t}^{s}$ such that $U^{s}(A)=u^{s}\left(t, m^{\prime \prime}\right)$, then $m^{\prime \prime} \geq l_{t}^{b}$. (ii) If there are $m^{\prime}, m^{\prime \prime} \in \mathbb{R}$ such that $m^{\prime} \leq \bar{m}_{t}^{b}, U^{b}(A)=u^{b}\left(t, m^{\prime}\right)$ and $U^{s}(A)=u^{s}\left(t, m^{\prime \prime}\right)$, then $m^{\prime} \leq m^{\prime \prime}$.

For (i), by contradiction, suppose that $m^{\prime \prime}<l_{t}^{b}$. Define $m=\left(m^{\prime \prime}+l_{t}^{b}\right) / 2$ and consider the contract $c=(t, m) \in \bar{Y}$. Then from $m^{\prime \prime}<l_{t}^{b}$ it follows that $m^{\prime \prime}<m$ and $l_{t}^{b}>m$. We have that $U^{b}(A)<u^{b}\left(t, l_{t}^{b}\right)<u^{b}(t, m)$ and $U^{s}(A)=u^{s}\left(t, m^{\prime \prime}\right)<u^{s}(t, m)$, thereby 
contradicting that $A$ satisfies Condition (ii) of Definition 3.1.

For (ii), by contradiction, suppose that $m^{\prime}>m^{\prime \prime}$. Define $m=\left(m^{\prime}+m^{\prime \prime}\right) / 2$ and consider the contract $c=(t, m) \in \bar{Y}$. Then from $m^{\prime}>m^{\prime \prime}$, it follows that $m^{\prime}>m$ and $m^{\prime \prime}<m$. We have that $U^{b}(A)=u^{b}\left(t, m^{\prime}\right)<u^{b}(t, m)$ and $U^{s}(A)=u^{s}\left(t, m^{\prime \prime}\right)<u^{s}(t, m)$, contradicting that $A$ satisfies Condition (ii) of Definition 3.1.

Step 2: The construction of $(p, q) \in \mathbb{R}^{T} \times\{0,1\}^{T}$.

For each $c=(t, m) \in A$, we define $p_{t}=m$ and $q_{t}=0$. For every $t \in T \backslash \tau(A)$, we define $p$ and $q$ as in Table 1.

Table 1 Construction of $\left(p_{t}, q_{t}\right)$ for $t \in T \backslash \tau(A)$.

\begin{tabular}{ccc}
\hline & $(s-1)$ & $(s-2)$ \\
\hline$(b-1)$ & $p_{t}=0, q_{t}=0$ & $p_{t}=m^{\prime \prime}, q_{t}=0$ \\
\hline$(b-2)$ & $p_{t}=m^{\prime}, q_{t}=0$ & $p_{t}=m^{\prime \prime}, q_{t}=0$ \\
\hline$(b-3)$ & $p_{t}=l_{t}^{b}, q_{t}=1$ & $p_{t}=l_{t}^{b}, q_{t}=1$ \\
\hline
\end{tabular}

Step 3: $(p, q, A)$ is a QCCE.

Let some $b \in B$ be given. Consider $t \in T^{b}$ such that $q_{t}=0$. We are either in Case $(b-1)$ or in Case $(b-2)$. If $(b-1)$ occurs, it is obvious that $U^{b}(A)>u^{b}\left(t, p_{t}\right)$. If $(b-2)$ occurs, by $(s-1)$ and statement (ii) of Step 1 for $(s-2)$, we have that $U^{b}(A) \geq u^{b}\left(t, p_{t}\right)$. We conclude that $A^{b} \in \delta^{b}(p, q)$, so Condition (i) of Definition 4.1 holds.

Let some $s \in S$ be given. Consider $t \in T^{s}$. If $(s-1)$ occurs, then it is obvious that $U^{s}(A)>u^{s}\left(t, p_{t}\right)$. If $(s-2)$ occurs, by $(b-1),(b-2)$, and statement (i) of Step 1 for $(b-3)$, we have that $U^{s}(A) \geq u^{s}\left(t, p_{t}\right)$. Thus, $A^{s} \in \delta^{s}(p)$ and so Condition (ii) of Definition 4.1 holds.

For each $t \in T$ such that $q_{t}=1$, by Table $1, p_{t}=l_{t}^{\beta(t)}$, so Condition (iii) of Definition 4.1 holds.

We have shown that $(p, q, A)$ is a QCCE.

Q.E.D.

\section{A.4 Proof of Theorem 5.1}

Proposition 4.3 implies $\mathcal{A}^{\mathrm{SO}}(\mathcal{E}) \subseteq \mathcal{A}^{\mathrm{QCCE}}(\mathcal{E})$. In the following, we show $\mathcal{A}^{\mathrm{QCCE}}(\mathcal{E}) \subseteq$ $\mathcal{A}^{\mathrm{SO}}(\mathcal{E})$. Let $A \in \mathcal{A}^{\mathrm{QCCE}}(\mathcal{E})$ and let $(p, q, A)$ be a QCCE for $\mathcal{E}$. Evidently, $A$ is an outcome. The following shows that $A$ is stable.

Step 1: $A$ satisfies Condition (i) of Definition 3.1.

For each $i \in B \cup S$ such that $A^{i}=\emptyset$, it is straightforward that $A^{i} \in \mathrm{Ch}^{i}(A)$.

For each $i \in B \cup S$ such that $A^{i} \neq \emptyset$, to show $A^{i} \in \mathrm{Ch}^{i}(A)$, by contradiction, suppose there is some $i \in B \cup S$ such that $A^{i} \notin \mathrm{Ch}^{i}(A)$, so $U^{i}(A)<U^{i}(\emptyset)$. If $i \in B$ then it follows that $A^{i} \notin \delta^{b}(p, q)$ and if $i \in S$ it follows that $A^{i} \notin \delta^{s}(p)$, contradicting that $(p, q, A)$ is a QCCE. Consequently, for each $i \in B \cup S$ such that $A^{i} \neq \emptyset$, it holds that $A^{i} \in \operatorname{Ch}^{i}(A)$. 
Step 2: $A$ satisfies Condition (ii) of Definition 3.1.

By contradiction, suppose there is $c=(t, m) \in \bar{Y}$ such that $U^{\beta(t)}(\{c\})>U^{\beta(t)}(A)$ and $U^{\sigma(t)}(\{c\})>U^{\sigma(t)}(A)$.

Case 1: $m \geq p_{t}$.

Then $u^{\beta(t)}\left(t, p_{t}\right) \geq u^{\beta(t)}(t, m)=U^{\beta(t)}(\{c\})>U^{\beta(t)}(A)$. By Condition (i) of Definition 4.1, it holds that $A^{\beta(t)} \in \delta^{\beta(t)}(p, q)$, so $q_{t}=1$. By Condition (iii) of Definition 4.1, it follows that $p_{t} \geq l_{t}^{\beta(t)}$. Since $(t, m) \in \bar{Y}$, we have that $m \leq l_{t}^{\beta(t)}$ and so $p_{t}=l_{t}^{\beta(t)}=m$. It follows that $\{c\} \in \gamma^{s}(p)$. By $U^{\sigma(t)}(\{c\})>U^{\sigma(t)}(A)$, we have that $A^{\sigma(t)} \notin \delta^{\sigma(t)}(p)$, a contradiction to Condition (ii) of Definition 4.1.

Case 2: $m<p_{t}$.

It holds that $U^{\sigma(t)}(\{c\})>U^{\sigma(t)}(A) \geq u^{\sigma(t)}\left(t, p_{t}\right)$, so $m>p_{t}$, a contradiction.

Consequently, $A$ satisfies Condition (ii) of Definition 3.1.

Q.E.D.

\section{A.5 Proof of Proposition 5.5}

We only need to show that $\mathcal{C}(\mathcal{E})=\mathcal{A}^{\mathrm{SO}}(\mathcal{E})$. It then follows by Proposition 3.4 and Theorem 5.1 that $\mathcal{C}(\mathcal{E})=\mathcal{A}^{\mathrm{QCCE}}(\mathcal{E}) \neq \emptyset$.

Step 1: For each $A \in \mathcal{A}^{\mathrm{SO}}(\mathcal{E}), A \in \mathcal{C}(\mathcal{E})$.

By contradiction, suppose that there is $A \in \mathcal{A}^{\mathrm{SO}}(\mathcal{E})$ such that $A \notin \mathcal{C}(\mathcal{E})$. Then there is $A^{\prime} \in \mathcal{A}$ and $N \subseteq B \cup S$ such that:

(i) For every $i \in(B \cup S) \backslash N,\left(A^{\prime}\right)^{i}=\emptyset$.

(ii) For every $j \in N, U^{j}\left(A^{\prime}\right)>U^{j}(A)$.

By (ii) and since $A$ is a stable outcome, for each $j \in N, U^{j}\left(A^{\prime}\right)>U^{j}(A) \geq U^{j}(\emptyset)$ and so $\left(A^{\prime}\right)^{j} \neq \emptyset$. Let $k$ be the trading partner of $j$ in $A^{\prime}$. By (i) it holds that $k \notin(B \cup S) \backslash N$, so $k \in N$, and therefore $U^{k}\left(A^{\prime}\right)>U^{k}(A)$. Thus, Condition (ii) of Definition 3.1 is violated, contradicting that $A \in \mathcal{A}^{\mathrm{SO}}(\mathcal{E})$.

Step 2: For each $A \in \mathcal{C}(\mathcal{E}), A \in \mathcal{A}^{\mathrm{SO}}(\mathcal{E})$.

By contradiction, suppose that there is $A \in \mathcal{C}(\mathcal{E})$ such that $A \notin \mathcal{A}^{\mathrm{SO}}(\mathcal{E})$.

Case 1: Condition (i) of Definition 3.1 is violated.

Then there is $i \in B \cup S$ such that $U^{i}(\emptyset)>U^{i}(A)$. Take $A^{\prime}=\emptyset$ and $N=\{i\}$. Then $U^{i}\left(A^{\prime}\right)>U^{i}(A)$ implies that $A \notin \mathcal{C}(\mathcal{E})$, a contradiction.

Case 2: Condition (ii) of Definition 3.1 is violated.

Then there is $c=(t, m) \in \bar{Y}$ such that $U^{\beta(t)}(\{c\})>U^{\beta(t)}(A)$ and $U^{\sigma(t)}(\{c\})>U^{\sigma(t)}(A)$. Take $A^{\prime}=\{c\}$ and $N=\{\beta(t), \sigma(t)\}$. Now $U^{\beta(t)}\left(A^{\prime}\right)>U^{\beta(t)}(A)$ and $U^{\sigma(t)}\left(A^{\prime}\right)>U^{\sigma(t)}(A)$ imply that $A \notin \mathcal{C}(\mathcal{E})$, a contradiction.

Q.E.D.

\section{A.6 Proof of Proposition 5.7}

By Corollary 4.4, a QCCE exists. Let $(p, Q, A)$ be a QCCE and let $I_{1} \in \mathcal{T}^{S}$ be a set 
of $s_{1}$-equivalent trades. We construct a $\operatorname{QCCE}\left(p^{1}, q^{1}, A^{1}\right)$ such that, for every $t, t^{\prime} \in I_{1}$, $p_{t}^{1}=p_{t^{\prime}}^{1}$ and, for every $t \in T \backslash I_{1}, p_{t}^{1}=p_{t}$. Then, using the same reasoning, given $\left(p^{1}, q^{1}, A^{1}\right)$ and $I_{2} \in \mathcal{T}^{S} \backslash\left\{I_{1}\right\}$, we can derive a QCCE $\left(p^{2}, q^{2}, A^{2}\right)$ such that, for every $t, t^{\prime} \in I_{2}, p_{t}^{2}=p_{t^{\prime}}^{2}$ and, for every $t \in T \backslash I_{2}, p_{t}^{2}=p_{t}^{1}$, and so on. Since $\mathcal{T}^{S}$ contains finitely many, say $k$, sets, it follows that $\left(p^{k}, q^{k}, A^{k}\right)$ is a QCCE that meets the requirements of Proposition 5.7.

We define $\left(p^{1}, q^{1}, A^{1}\right)$ as follows:

(i) For every $t^{\prime} \in I_{1}, p_{t^{\prime}}^{1}=\max _{t \in I_{1}} p_{t}$ and, for every $t \in T \backslash I_{1}, p_{t}^{1}=p_{t}$.

(ii) $q^{1}=q$.

(iii) $A^{1}=A$.

To show that $\left(p^{1}, q^{1}, A^{1}\right)$ is a QCCE, we show first that, for every $(t, m) \in A^{1}, p_{t}^{1}=m$. Suppose not. Then there is $\left(t^{\prime}, m^{\prime}\right) \in A^{1}$ such that $t^{\prime} \in I_{1}$ and $p_{t^{\prime}}^{1}>m^{\prime}=p_{t^{\prime}}$. Let $t \in I_{1}$ be such that $p_{t^{\prime}}^{1}=p_{t}$. It holds that $\left\{\left(t, p_{t}\right)\right\} \in \gamma^{s_{1}}(p)$. Since $u^{s_{1}}(t, \cdot)=u^{s_{1}}\left(t^{\prime}, \cdot\right)$ and $p_{t}>$ $p_{t^{\prime}}$, it follows that $U^{s_{1}}\left(\left\{\left(t, p_{t}\right)\right\}\right)>U^{s_{1}}\left(\left\{\left(t^{\prime}, p_{t^{\prime}}\right)\right\}\right)$, a contradiction to $\left\{\left(t^{\prime}, p_{t^{\prime}}\right)\right\} \in \delta^{s_{1}}(p)$. Consequently, for every $(t, m) \in A^{1}, p_{t}^{1}=m$, so $p_{t}^{1}=p_{t},\{(t, m)\} \in \gamma^{\beta(t)}\left(p^{1}, q^{1}\right)$, and $\{(t, m)\} \in \gamma^{\sigma(t)}\left(p^{1}\right)$.

We show next that $\left(p^{1}, q^{1}, A^{1}\right)$ satisfies the conditions of Definition 4.1.

Step 1: Condition (i) of Definition 4.1 holds for each $b \in B$.

Let some $b \in B$ be given. It holds that $\left(A^{1}\right)^{b}=A^{b} \in \gamma^{b}\left(p^{1}, q^{1}\right)$. For every $t \in T^{b}$, since $p_{t}^{1} \geq p_{t}$ and $q^{1}=q$, it holds that $\left(A^{1}\right)^{b} \in \delta^{b}\left(p^{1}, q^{1}\right)$. Thus Condition (i) of Definition 4.1 holds for each $b \in B$.

We continue by showing that $\left(p^{1}, q^{1}, A^{1}\right)$ satisfies Condition (ii) of Definition 4.1.

Step 2: Condition (ii) of Definition 4.1 holds for each $s \in S$.

For each $s \in S \backslash\left\{s_{1}\right\}$, since $\left(A^{1}\right)^{s}=A^{s}$ and, for each $t \in T^{s}, p_{t}^{1}=p_{t}, A^{s} \in \delta^{s}(p)$ implies $\left(A^{1}\right)^{s} \in \delta^{s}\left(p^{1}\right)$. Thus Condition (ii) of Definition 4.1 holds for every $s \in S \backslash\left\{s_{1}\right\}$.

To show that $s_{1}$ satisfies Condition (ii) of Definition (4.1), we distinguish two cases.

Case 1: $A^{s_{1}}=\emptyset$. It holds that, for every $t \in I_{1}, U^{s_{1}}(\emptyset) \geq u^{s_{1}}\left(t, p_{t}\right)$. Using the fact that $I_{1}$ is a set of $s_{1}$-equivalent trades, it follows that, for every $t \in I_{1}, U^{s_{1}}(\emptyset) \geq u^{s_{1}}\left(t, \max _{t^{\prime} \in I_{1}} p_{t^{\prime}}\right)=$ $u^{s_{1}}\left(t, p_{t}^{1}\right)$. For each $t \in T^{s_{1}} \backslash I_{1}$, it holds that $p_{t}^{1}=p_{t}$, so $U^{s_{1}}(\emptyset) \geq u^{s_{1}}\left(t, p_{t}^{1}\right)$. Thus Condition (ii) of Definition 4.1 holds for $s_{1}$.

Case 2: $A^{s_{1}} \neq \emptyset$. It holds that, for every $t \in I_{1}, U^{s_{1}}(A) \geq u^{s_{1}}\left(t, p_{t}\right)$. Using the fact that $I_{1}$ is a set of $s_{1}$-equivalent trades, it follows that, for every $t \in I_{1}, U^{s_{1}}(A) \geq u^{s_{1}}\left(t, p_{t}^{1}\right)$. For each $t \in T^{s_{1}} \backslash I_{1}$, it holds that $p_{t}^{1}=p_{t}$, so $U^{s_{1}}(A) \geq u^{s_{1}}\left(t, \max _{t^{\prime} \in I_{1}} p_{t^{\prime}}\right)=u^{s_{1}}\left(t, p_{t}^{1}\right)$. Since $A^{1}=A$, we find that Condition (ii) of Definition 4.1 holds for $s_{1}$.

Step 3: It is easily seen that $\left(p^{1}, q^{1}, A^{1}\right)$ satisfies Condition (iii) of Definition 4.1.

Consequently, $\left(p^{1}, q^{1}, A^{1}\right)$ is a QCCE.

Q.E.D.

\section{A.7 Proof of Proposition 5.8}


By Corollary 4.4, a QCCE exists. Let $(p, q, A)$ be a QCCE and let $I_{1} \in \mathcal{T}^{B}$ be a set of $b_{1}$-equivalent trades. We derive a $\operatorname{QCCE}\left(p^{1}, q^{1}, A^{1}\right)$ such that, for every $t, t^{\prime} \in I_{1}, p_{t}^{1}=p_{t^{\prime}}^{1}$ and, for every $t \in T \backslash I_{1}, p_{t}^{1}=p_{t}$. Then, using the same reasoning, given $\left(p^{1}, q^{1}, A^{1}\right)$ and $I_{2} \in \mathcal{T}^{B} \backslash I_{1}$, we can derive a QCCE $\left(p^{2}, q^{2}, A^{2}\right)$ such that, for every $t, t^{\prime} \in I_{2}, p_{t}^{2}=p_{t^{\prime}}^{2}$ and, for every $t \in T \backslash I_{2}, p_{t}^{2}=p_{t}^{1}$, and so on. Since $\mathcal{T}^{B}$ contains finitely many, say $k$, sets, it follows that $\left(p^{k}, q^{k}, A^{k}\right)$ is a QCCE that meets the requirements of Proposition 5.8.

We define $\left(p^{1}, q^{1}, A^{1}\right)$ as follows.

(i) For every $t^{\prime} \in I_{1}, p_{t^{\prime}}^{1}=\min _{t \in I_{1}} p_{t}$ and, for every $t \in T \backslash I_{1}, p_{t}^{1}=p_{t}$.

(ii) For every $t \in I_{1}$ such that $p_{t}>l_{t}^{b_{1}}$ and $p_{t}^{1}=l_{t}^{b_{1}}, q_{t}^{1}=1$. For every $t \in I_{1}$ such that $q_{t}=1$ and $p_{t}^{1}<l_{t}^{b_{1}}, q_{t}^{1}=0$. Otherwise, $q_{t}^{1}=q_{t}$.

(iii) $A^{1}=A$.

To show that $\left(p^{1}, q^{1}, A^{1}\right)$ is a QCCE, we show first that, for every $(t, m) \in A^{1}, p_{t}^{1}=m$. Suppose not. Then there is $\left(t^{\prime}, m^{\prime}\right) \in A^{1}$ such that $t^{\prime} \in I_{1}$ and $p_{t^{\prime}}^{1}<m^{\prime}=p_{t^{\prime}}$. Let $t \in I_{1}$ be such that $p_{t^{\prime}}^{1}=p_{t}$. Since $\left(t^{\prime}, p_{t^{\prime}}\right) \in A$ and $I_{1}$ is a set of $b_{1}$-equivalent trades, it holds that $p_{t^{\prime}} \leq l_{t^{\prime}}^{b_{1}}=l_{t}^{b_{1}}$, so $p_{t}<l_{t}^{b_{1}}$ and $q_{t}=0$. It follows that $\left\{\left(t, p_{t}\right)\right\} \in \gamma^{b_{1}}(p, q)$. Since $u^{b_{1}}(t, \cdot)=u^{b_{1}}\left(t^{\prime}, \cdot\right)$ and $p_{t}<p_{t^{\prime}}$, it follows that $U^{b_{1}}\left(\left\{\left(t, p_{t}\right)\right\}\right)>U^{b_{1}}\left(\left\{\left(t^{\prime}, p_{t^{\prime}}\right)\right\}\right)$, a contradiction to $\left\{\left(t^{\prime}, p_{t^{\prime}}\right)\right\} \in \delta^{b_{1}}(p, q)$. Consequently, for every $(t, m) \in A^{1}, p_{t}^{1}=m$, so $p_{t}^{1}=p_{t}, q_{t}^{1}=q_{t},\{(t, m)\} \in \gamma^{\beta(t)}\left(p^{1}, q^{1}\right)$, and $\{(t, m)\} \in \gamma^{\sigma(t)}\left(p^{1}\right)$.

We show next that $\left(p^{1}, q^{1}, A^{1}\right)$ satisfies the conditions of Definition 4.1.

Step 1: Condition (i) of Definition 4.1 holds for each $b \in B$.

For every $b \in B \backslash\left\{b_{1}\right\}$, for every $t \in T^{b}, p_{t}^{1}=p_{t}$ and $q_{t}^{1}=q_{t}$, so $\left(A^{1}\right)^{b}=A^{b} \in \delta^{b}(p, q)=$ $\delta^{b}\left(p^{1}, q^{1}\right)$.

To show that $b_{1}$ satisfies Condition (i) of Definition 4.1, we distinguish three cases.

Case 1: $\tau\left(A^{b_{1}}\right) \cap I_{1} \neq \emptyset$. Denote the element in $\tau\left(A^{b_{1}}\right) \cap I_{1}$ by $t^{\prime}$. We have already shown that $p_{t^{\prime}}^{1}=p_{t^{\prime}}=\min _{t \in I_{1}} p_{t}$, so, for every $t \in I_{1}, U^{b_{1}}(A) \geq u^{b_{1}}\left(t, p_{t}^{1}\right)$. Since for every $t \in T^{b_{1}} \backslash I_{1}$, $p_{t}^{1}=p_{t}$ and $q_{t}^{1}=q_{t}$, we have $\left(A^{1}\right)^{b_{1}}=A^{b_{1}} \in \delta^{b_{1}}\left(p^{1}, q^{1}\right)$.

Case 2: $\tau\left(A^{b_{1}}\right) \cap I_{1}=\emptyset$ and there is $t \in I_{1}$ such that $\left\{\left(t, p_{t}\right)\right\} \in \gamma^{b_{1}}(p, q)$. For every $t \in I_{1}$ such that $\left\{\left(t, p_{t}\right)\right\} \in \gamma^{b_{1}}(p, q)$, it holds that $U^{b_{1}}(A) \geq u^{b_{1}}\left(t, p_{t}\right)$. Let $m=$ $\min _{\left\{t \in I_{1} \mid\left\{\left(t, p_{t}\right)\right\} \in \gamma^{\left.b_{1}(p, q)\right\}}\right.} p_{t}$. Since $I_{1}$ is a set of $b_{1}$-equivalent trades, we have, for every $t \in I_{1}$ such that $\left\{\left(t, p_{t}\right)\right\} \in \gamma^{b_{1}}(p, q), U^{b_{1}}(A) \geq u^{b_{1}}(t, m)$. For every $t \in I_{1}$ such that $\left\{\left(t, p_{t}\right)\right\} \notin$ $\gamma^{b_{1}}(p, q)$, we have $p_{t} \geq l_{t}^{b_{1}} \geq m$. It follows that $m=\min _{t \in I_{1}} p_{t}=p_{t}^{1}$, so, for every $t \in I_{1}, U^{b_{1}}(A) \geq u^{b_{1}}\left(t, p_{t}^{1}\right)$. Since for each $t \in T^{b_{1}} \backslash I_{1}, p_{t}^{1}=p_{t}$ and $q_{t}^{1}=q_{t}$, it holds that $\left(A^{1}\right)^{b_{1}}=A^{b_{1}} \in \delta^{b_{1}}\left(p^{1}, q^{1}\right)$.

Case 3: $\tau\left(A^{b_{1}}\right) \cap I_{1}=\emptyset$ and, for every $t \in I_{1},\left\{\left(t, p_{t}\right)\right\} \notin \gamma^{b_{1}}(p, q)$. It holds that, for every $t \in I_{1}, p_{t} \geq l_{t}^{b_{1}}$. If for each $t \in I_{1}, p_{t}>l_{t}^{b_{1}}$, then since $I_{1}$ is a set of $b_{1}$-equivalent trades, we have, for every $t^{\prime} \in I_{1}, p_{t^{\prime}}=\min _{t \in I_{1}} p_{t}>l_{t^{\prime}}^{b_{1}}$. Since, for every $t \in T^{b_{1}} \backslash I_{1}, p_{t}^{1}=p_{t}$ and $q_{t}^{1}=q_{t}$, it follows that $\left(A^{1}\right)^{b_{1}}=A^{b_{1}} \in \delta^{b_{1}}\left(p^{1}, q^{1}\right)$. 
If there is $t \in I_{1}$ such that $p_{t}=l_{t}^{b_{1}}$, then it follows from $\left\{\left(t, p_{t}\right)\right\} \notin \gamma^{b_{1}}(p, q)$ that $q_{t}=1$. Since $I_{1}$ is a set of $b_{1}$-equivalent trades, it follows that $l_{t}^{b_{1}}=\min _{t \in I_{1}} p_{t}$. Note that, for every $t \in I_{1}$, if $p_{t}>l_{t}^{b_{1}}$ and $p_{t}^{1}=l_{t}^{b_{1}}$, then $q_{t}^{1}=1$. Thus, for every $t \in I_{1}$, it holds that $\left\{\left(t, p_{t}^{1}\right)\right\} \notin \gamma^{b_{1}}\left(p^{1}, q^{1}\right)$. Since for each $t \in T^{b_{1}} \backslash I_{1}, p_{t}^{1}=p_{t}$ and $q_{t}^{1}=q_{t}$, we have that $\left(A^{1}\right)^{b_{1}}=A^{b_{1}} \in \delta^{b_{1}}\left(p^{1}, q^{1}\right)$.

Step 2: Condition (ii) of Definition 4.1 holds for each $s \in S$.

Since, for every $s \in S, A^{s} \in \gamma^{s}\left(p^{1}\right)$ and $p^{1} \leq p$, we have that $A^{s} \in \delta^{s}\left(p^{1}\right)$.

Step 3: It is easily seen that $\left(p^{1}, q^{1}, A^{1}\right)$ satisfies Condition (iii) of Definition 4.1.

Consequently, $\left(p^{1}, q^{1}, A^{1}\right)$ is a QCCE.

Q.E.D.

\section{A.8 Proof of Theorem 5.10}

For every $b \in B$, for every $\ell \in L$, define $I_{b, \ell}=\{b\} \times S \times\{\ell\}$. It holds that all trades in $I_{b, \ell}$ are $b$-equivalent. By Proposition 5.8, there is a QCCE $(p, q, A)$ with buyer-uniform prices, so for every $t, t^{\prime} \in I_{b, \ell}$ it holds that $p_{t}=p_{t^{\prime}}$. We denote this price by $p_{b, \ell}$. Next, for every $\ell \in L$, we define $p_{\ell}=\max _{b \in B} p_{b, \ell}$. Let $\bar{p} \in \mathbb{R}^{T}$ be defined by

$$
\bar{p}_{t}=p_{\lambda(t)}, \quad t \in T
$$

We argue that $(\bar{p}, q, A)$ is a QCCE with uniform prices.

To show that $(\bar{p}, q, A)$ is a QCCE, we show first that, for every $(t, m) \in A, \bar{p}_{t}=m$. Suppose not. Then there is $\left(t^{\prime}, m^{\prime}\right)=\left(\left(b^{\prime}, s^{\prime}, \ell^{\prime}\right), m^{\prime}\right) \in A$ such that $\bar{p}_{t^{\prime}}>m^{\prime}=p_{t^{\prime}}$. Let $b \in B$ be such that $\bar{p}_{t^{\prime}}=p_{b, \ell^{\prime}}$. Since the prices $p$ are buyer-uniform, for $t=\left(b, s^{\prime}, \ell^{\prime}\right)$ it holds that $p_{t}=\bar{p}_{t^{\prime}}>p_{t^{\prime}}$. It holds that $\left\{\left(t, p_{t}\right)\right\} \in \gamma^{s^{\prime}}(p)$ and $U^{s^{\prime}}\left(\left\{\left(t, p_{t}\right)\right\}\right)>U^{s^{\prime}}\left(\left\{\left(t^{\prime}, p_{t^{\prime}}\right)\right\}\right)$, a contradiction to $\left\{\left(t^{\prime}, p_{t^{\prime}}\right)\right\} \in \delta^{s^{\prime}}(p)$. Consequently, for every $(t, m) \in A, \bar{p}_{t}=m$, so $\bar{p}_{t}=p_{t}$, $\{(t, m)\} \in \gamma^{\beta(t)}(\bar{p}, q)$, and $\{(t, m)\} \in \gamma^{\sigma(t)}(\bar{p})$.

We show next that $(\bar{p}, q, A)$ satisfies Condition (i) of Definition 4.1. Let some $b \in B$ be given. We have already shown that $A^{b} \in \gamma^{b}(\bar{p}, q)$. Since $A^{b} \in \delta^{b}(p, q)$ and $\bar{p} \geq p$, it follows that $A^{b} \in \delta^{b}(\bar{p}, q)$.

We show next that $(\bar{p}, q, A)$ satisfies Condition (ii) of Definition 4.1. Let some $s \in S$ be given. We have already shown that $A^{s} \in \gamma^{s}(\bar{p})$. From $A^{s} \in \delta^{s}(p)$, it follows that $U^{s}\left(A^{s}\right) \geq$ $U^{s}(\emptyset)$. Suppose there is $\left\{\left(t, \bar{p}_{t}\right)\right\} \in \gamma^{s}(\bar{p})$ such that $u^{s}\left(t, \bar{p}_{t}\right)>U^{s}(A)$. From $A^{s} \in \delta^{s}(p)$, it follows that $\bar{p}_{t}>p_{t}$. Let $b \in B$ be such that $\bar{p}_{t}=p_{(b, s, \lambda(t))}$. Then $u^{s}\left((b, s, \lambda(t)), p_{(b, s, \lambda(t))}\right)=$ $u^{s}\left(t, \bar{p}_{t}\right)>U^{s}\left(A^{s}\right)$, a contradiction to $A^{s} \in \delta^{s}(p)$. Consequently, it holds that $A^{s} \in \delta^{s}(\bar{p})$.

We complete the proof by showing that $(\bar{p}, q, A)$ satisfies Condition (iii) of Definition 4.1. This follows easily from the fact that $(p, q, A)$ satisfies Condition (iii) of Definition 4.1 and $\bar{p} \geq p$.

Q.E.D.

\section{A.9 Proof of Theorem 5.12}


Let $B_{1}, B_{2}$, and $B_{3}$ be a partition of $B$ such that

$$
\begin{aligned}
& B_{1}=\left\{b \in B: U^{b}(A)>U^{b}\left(A^{\prime}\right)\right\}, \\
& B_{2}=\left\{b \in B: U^{b}\left(A^{\prime}\right)>U^{b}(A)\right\}, \\
& B_{3}=\left\{b \in B: U^{b}(A)=U^{b}\left(A^{\prime}\right)\right\} .
\end{aligned}
$$

Let $S_{1}, S_{2}$, and $S_{3}$ be a partition of $S$ such that

$$
\begin{aligned}
& S_{1}=\left\{s \in S: U^{s}\left(A^{\prime}\right)>U^{s}(A)\right\}, \\
& S_{2}=\left\{s \in S: U^{s}(A)>U^{s}\left(A^{\prime}\right)\right\}, \\
& S_{3}=\left\{s \in S: U^{s}(A)=U^{s}\left(A^{\prime}\right)\right\} .
\end{aligned}
$$

Let some $b \in B_{1}$ be given. We have that $U^{b}(A)>U^{b}\left(A^{\prime}\right)$, so $A^{b} \neq \emptyset$. Let $t$ be the trade corresponding to $A^{b}$ and $s=\sigma(t)$. We show that $s \in S_{1}$ by distinguishing two cases.

Case 1: $\left\{\left(t, p_{t}^{\prime}\right)\right\} \in \gamma^{b}\left(p^{\prime}, q\right)$. It holds by $U^{b}(A)>U^{b}\left(A^{\prime}\right)$ that $p_{t}<p_{t}^{\prime}$. Since $U^{s}\left(A^{\prime}\right) \geq$ $u^{s}\left(t, p_{t}^{\prime}\right)$, it holds that $U^{s}\left(A^{\prime}\right)>u^{s}\left(t, p_{t}\right)=U^{s}(A)$, so $s \in S_{1}$.

Case 2: $\left\{\left(t, p_{t}^{\prime}\right)\right\} \notin \gamma^{b}\left(p^{\prime}, q\right)$. Since $q$ remains the same at the two QCCEs, it holds that $p_{t}^{\prime}>l_{t}^{b}$ and $p_{t} \leq l_{t}^{b}$. Since $U^{s}\left(A^{\prime}\right) \geq u^{s}\left(t, p_{t}^{\prime}\right)$, it follows that $U^{s}\left(A^{\prime}\right)>u^{s}\left(t, p_{t}\right)=U^{s}(A)$, so $s \in S_{1}$.

We conclude that $\left|B_{1}\right| \leq\left|S_{1}\right|$.

Let some $s \in S_{1}$ be given. We have that $U^{s}\left(A^{\prime}\right)>U^{s}(A)$, so $\left(A^{\prime}\right)^{s} \neq \emptyset$. Let $t$ be the trade related to $\left(A^{\prime}\right)^{s}$ and $b=\beta(t)$. Since $u^{s}\left(t, p_{t}^{\prime}\right)=U^{s}\left(A^{\prime}\right)>U^{s}(A) \geq u^{s}\left(t, p_{t}\right)$, we have that $p_{t}^{\prime}>p_{t}$. From $\left\{\left(t, p_{t}^{\prime}\right)\right\} \in \gamma^{b}\left(p^{\prime}, q\right)$ and $p_{t}^{\prime}>p_{t}$, it follows that $\left\{\left(t, p_{t}\right)\right\} \in \gamma^{b}(p, q)$. Thus, we have $U^{b}(A) \geq u^{b}\left(t, p_{t}\right)>u^{b}\left(t, p_{t}^{\prime}\right)=U^{b}\left(A^{\prime}\right)$, so $b \in B_{1}$.

The result of the previous paragraph implies that $\left|S_{1}\right| \leq\left|B_{1}\right|$. Together with $\left|B_{1}\right| \leq\left|S_{1}\right|$, this implies that $\left|B_{1}\right|=\left|S_{1}\right|$. Moreover, it is easily seen that agents in $B_{1}$ are matched with agents in $S_{1}$ at two QCCEs. A similar argument can be used to show that $\left|B_{2}\right|=\left|S_{2}\right|$ and agents in $B_{2}$ are matched with agents in $S_{2}$ at the two QCCEs.

By the above analysis, for every $b \in B_{1}$ and $t \in \tau\left(A^{b}\right)$, for every $b^{\prime} \in B_{2} \cup B_{3}$ and $t^{\prime} \in \tau\left(\left(A^{\prime}\right)^{b^{\prime}}\right)$, it holds that $\sigma(t) \neq \sigma\left(t^{\prime}\right)$. Since $A$ is an outcome, it holds that, for every $b \in B_{1}$ and $t \in \tau\left(A^{b}\right)$, for every $b^{\prime} \in B_{1}$ and $t^{\prime} \in \tau\left(A^{b^{\prime}}\right), b \neq b^{\prime}$ implies $\sigma(t) \neq \sigma\left(t^{\prime}\right)$. Since $A^{\prime}$ is an outcome, it holds that, for every $b \in B_{2} \cup B_{3}$ and $t \in \tau\left(\left(A^{\prime}\right)^{b}\right)$, for every $b^{\prime} \in B_{2} \cup B_{3}$ and $t^{\prime} \in \tau\left(\left(A^{\prime}\right)^{b^{\prime}}\right), b \neq b^{\prime}$ implies $\sigma(t) \neq \sigma\left(t^{\prime}\right)$. It follows that $A \wedge A^{\prime}$ is an outcome. By a similar argument it can be shown that $A \vee A^{\prime}$ is an outcome.

Denote $p \wedge p^{\prime}$ by $\underline{p}$ and $A \wedge A^{\prime}$ by $\underline{A}$. We show next that $(\underline{p}, q, \underline{A})$ is a QCCE.

Step 1: Condition (i) of Definition 4.1 holds.

Let some $b \in B_{1}$ be given. It holds that $\underline{A}^{b}=A^{b}$. Since $U^{b}(A)>U^{b}\left(A^{\prime}\right)$, it follows that $A^{b} \neq \emptyset$. Let $(t, m) \in T \times \mathbb{R}$ be such that $A^{b}=\{(t, m)\}$. From $U^{b}(A)>U^{b}\left(A^{\prime}\right)$ and the analysis of Cases 1 and 2 above, it follows that $\underline{p}_{t}=p_{t}<p_{t}^{\prime}$. Since $A^{b} \in \gamma^{b}(p, q)$, it 
holds that $m=p_{t}$, so $A^{b} \in \gamma^{b}(p, q)$. Obviously, it is true that $U^{b}(A) \geq U^{b}(\emptyset)$. For every $\{c\} \in \gamma^{b}(\underline{p}, q)$ such that $\mu(c)=p_{\tau(c)}$, it holds that $\{c\} \in \gamma^{b}(p, q)$, so $u^{b}(c) \leq U^{b}(A)$. For every $\{c\} \in \gamma^{b}(\underline{p}, q)$ such that $\mu(c)=p_{\tau(c)}^{\prime}$, it holds that $\{c\} \in \gamma^{b}\left(p^{\prime}, q\right)$, so $u^{b}(c) \leq U^{b}\left(A^{\prime}\right)<$ $U^{b}(A)$. Thus Condition (i) of Definition 4.1 holds for every $b \in B_{1}$.

Let some $b \in B_{2} \cup B_{3}$ be given. It holds that $\underline{A}^{b}=\left(A^{\prime}\right)^{b}$. If $\left(A^{\prime}\right)^{b}=\emptyset$, then clearly $\left(A^{\prime}\right)^{b} \in \gamma^{b}(p, q)$. Otherwise, let $(t, m) \in T \times \mathbb{R}$ be such that $\left(A^{\prime}\right)^{b}=\{(t, m)\}$. Since $b$ is matched with some $\sigma(t) \in S_{2} \cup S_{3}$ and by $U^{\sigma(t)}\left(A^{\prime}\right) \leq U^{\sigma(t)}(A)$, it holds that $\underline{p}_{t}=p_{t}^{\prime} \leq p_{t}$. Since $\left(A^{\prime}\right)^{b} \in \gamma^{b}\left(p^{\prime}, q\right)$, it holds that $m=p_{t}^{\prime}$, so $\left(A^{\prime}\right)^{b} \in \gamma^{b}(\underline{p}, q)$. Obviously, it is true that $U^{b}\left(A^{\prime}\right) \geq U^{b}(\emptyset)$. For every $\{c\} \in \gamma^{b}(\underline{p}, q)$ such that $\mu(c)=p_{\tau(c)}$, it holds that $\{c\} \in \gamma^{b}(p, q)$, so $u^{b}(c) \leq U^{b}(A) \leq U^{b}\left(A^{\prime}\right)$. For every $\{c\} \in \gamma^{b}(\underline{p}, q)$ such that $\mu(c)=p_{\tau(c)}^{\prime}$, it holds that $\{c\} \in \gamma^{b}\left(p^{\prime}, q\right)$, so $u^{b}(c) \leq U^{b}\left(A^{\prime}\right)$. Thus Condition (i) of Definition 4.1 holds for every $b \in B_{2} \cup B_{3}$.

Step 2: Condition (ii) of Definition 4.1 holds.

Let some $s \in S_{1}$ be given. By the first part of the proof it holds that there is $t \in \tau(A)$ such that $\sigma(t)=s$ and $\beta(t) \in B_{1}$. We have that $\underline{A}^{s}=A^{s}$. Moreover, from $U^{s}\left(A^{\prime}\right)>U^{s}(A)$, it follows that $\underline{p}_{t}=p_{t}<p_{t}^{\prime}$, so $A^{s} \in \gamma^{s}(p)$. Obviously, it is true that $U^{s}(A) \geq U^{s}(\emptyset)$. For every $\{c\} \in \gamma^{s}(\underline{p})$, since $A^{s} \in \delta^{s}(p)$ and $\underline{p}_{\tau(c)} \leq p_{\tau(c)}$, we have that $U^{s}(A) \geq u^{s}(c)$. It follows that Condition (ii) of Definition 4.1 holds for every $s \in S_{1}$.

Let some $s \in S_{2} \cup S_{3}$ be given. By the first part of the proof it holds that $\underline{A}^{s}=\left(A^{\prime}\right)^{s}$. If $\left(A^{\prime}\right)^{s}=\emptyset$, then clearly $\left(A^{\prime}\right)^{s} \in \gamma^{s}(\underline{p})$. Otherwise, there is $(t, m) \in \bar{Y}$ such that $\left(A^{\prime}\right)^{s}=$ $\{(t, m)\}$. From $U^{s}(A) \geq U^{s}\left(A^{\prime}\right)$, it follows that $\underline{p}_{t}=p_{t}^{\prime} \leq p_{t}$, so $\left(A^{\prime}\right)^{s} \in \gamma^{s}(\underline{p})$. Obviously, it is true that $U^{s}\left(A^{\prime}\right) \geq U^{s}(\emptyset)$. For every $\{c\} \in \gamma^{s}(\underline{p})$, since $\left(A^{\prime}\right)^{s} \in \delta^{s}\left(p^{\prime}\right)$ and $\underline{p}_{\tau(c)} \leq p_{\tau(c)}^{\prime}$, we have that $U^{s}\left(A^{\prime}\right) \geq u^{s}(c)$. It follows that Condition (ii) of Definition 4.1 holds for every $s \in S_{2} \cup S_{3}$.

Step 3: Condition (iii) of Definition 4.1 holds.

For each $t \in T$, if $\underline{p}_{t}<l_{t}^{\beta(t)}$, then $p_{t}<l_{t}^{\beta(t)}$ or $p_{t}^{\prime}<l_{t}^{\beta(t)}$, so since both $(p, q, A)$ and $\left(p^{\prime}, q, A^{\prime}\right)$ are QCCEs, it follows that $q_{t}=0$.

We conclude that $(\underline{p}, q, \underline{A})$ is a QCCE.

Denote $p \vee p^{\prime}$ by $\bar{p}$ and $A \vee A^{\prime}$ by $\bar{A}$.

We show next that $(\bar{p}, q, \bar{A})$ is a $\mathrm{QCCE}$.

Step 1: Condition (i) of Definition 4.1 holds.

Let some $b \in B_{1}$ be given. It holds that $\bar{A}^{b}=\left(A^{\prime}\right)^{b}$. Let $(t, m) \in \bar{Y}$ be such that $\left(A^{\prime}\right)^{b}=\{(t, m)\}$. Since $b$ is matched with some $\sigma(t) \in S_{1}$ and by $U^{\sigma(t)}(A)<U^{\sigma(t)}\left(A^{\prime}\right)$, it follows that $p_{t}<p_{t}^{\prime}=\bar{p}_{t}$. The fact that $\left(A^{\prime}\right)^{b} \in \gamma^{b}\left(p^{\prime}, q\right)$ now implies that $\left(A^{\prime}\right)^{b} \in \gamma^{b}(\bar{p}, q)$. Obviously, it is true that $U^{b}\left(A^{\prime}\right) \geq U^{b}(\emptyset)$. For every $\{c\} \in \gamma^{b}(\bar{p}, q)$ such that $\mu(c)=p_{\tau(c)}$, it holds that $\left\{\left(\tau(c), p_{\tau(c)}^{\prime}\right)\right\} \in \gamma^{b}\left(p^{\prime}, q\right)$, so $u^{b}(c) \leq u^{b}\left(\tau(c), p_{\tau(c)}^{\prime}\right) \leq U^{b}\left(A^{\prime}\right)$. For every $\{c\} \in$ $\gamma^{b}(\bar{p}, q)$ such that $\mu(c)=p_{\tau(c)}^{\prime}$, it holds that $\{c\} \in \gamma^{b}\left(p^{\prime}, q\right)$, so $u^{b}(c) \leq U^{b}\left(A^{\prime}\right)$. Thus 
Condition (i) of Definition 4.1 holds for every $b \in B_{1}$.

Let some $b \in B_{2} \cup B_{3}$ be given. It holds that $\bar{A}^{b}=A^{b}$. If $A^{b}=\emptyset$, then we clearly have $A^{b} \in \gamma^{b}(\bar{p}, q)$. Otherwise, let $(t, m) \in \bar{Y}$ be such that $A^{b}=\{(t, m)\}$. Since $b$ is matched with some agent $\sigma(t) \in S_{2} \cup S_{3}$ and by $U^{\sigma(t)}\left(A^{\prime}\right) \leq U^{\sigma(t)}(A)$, it holds that $p_{t}^{\prime} \leq p_{t}=\bar{p}_{t}$. From $A^{b} \in \gamma^{b}(p, q)$ it now follows that $A^{b} \in \gamma^{b}(\bar{p}, q)$. Obviously, it is true that $U^{b}(A) \geq U^{b}(\emptyset)$. For every $\{c\} \in \gamma^{b}(\bar{p}, q)$ such that $\mu(c)=p_{\tau(c)}$, it holds that $\{c\} \in \gamma^{b}(p, q)$, so $u^{b}(c) \leq U^{b}(A)$. For every $\{c\} \in \gamma^{b}(\bar{p}, q)$ such that $\mu(c)=p_{\tau(c)}^{\prime}$, it holds that $\left\{\left(\tau(c), p_{\tau(c)}\right)\right\} \in \gamma^{b}(p, q)$, so $u^{b}(c) \leq u^{b}\left(\tau(c), p_{\tau(c)}\right) \leq U^{b}(A)$. Thus Condition (i) of Definition 4.1 holds for every $b \in B_{2} \cup B_{3}$.

Step 2: Condition (ii) of Definition 4.1 holds.

Let some $s \in S_{1}$ be given. By the first part of the proof it holds that there is $t \in \tau(A)$ such that $\sigma(t)=s$ and $\beta(t) \in B_{1}$, so $U^{b}(A)>U^{b}\left(A^{\prime}\right)$. We have that $\bar{A}^{s}=\left(A^{\prime}\right)^{s}$. Moreover, from $U^{s}\left(A^{\prime}\right)>U^{s}(A)$, it follows that $p_{t}<p_{t}^{\prime}=\bar{p}_{t}$, so $\left(A^{\prime}\right)^{s} \in \gamma^{s}(\bar{p})$. Obviously, it is true that $U^{s}\left(A^{\prime}\right) \geq U^{s}(\emptyset)$. For every $\{c\} \in \gamma^{s}(\bar{p})$ such that $\mu(c)=p_{\tau(c)}$ it holds that $\{c\} \in \gamma^{s}(p)$, so $u^{s}(c) \leq U^{s}(A)<U^{s}\left(A^{\prime}\right)$. For every $\{c\} \in \gamma^{s}(\bar{p})$ such that $\mu(c)=p_{\tau(c)}^{\prime}$ it holds that $\{c\} \in \gamma^{s}\left(p^{\prime}\right)$, so $u^{s}(c) \leq U^{s}\left(A^{\prime}\right)$. It follows that Condition (ii) of Definition 4.1 holds for every $s \in S_{1}$.

Let some $s \in S_{2} \cup S_{3}$ be given. By the first part of the proof it holds that $\bar{A}^{s}=A^{s}$. If $A^{s}=\emptyset$, then clearly $A^{s} \in \gamma_{s}(\bar{p})$. Otherwise, there is $(t, m) \in \bar{Y}$ such that $A^{s}=\{(t, m)\}$. From $U^{s}(A) \geq U^{s}\left(A^{\prime}\right)$, it follows that $p_{t}^{\prime} \leq p_{t}=\bar{p}_{t}$, so $A^{s} \in \gamma^{s}(\bar{p})$. Obviously, it is true that $U^{s}(A) \geq U^{s}(\emptyset)$. For every $\{c\} \in \gamma^{s}(\bar{p})$ such that $\mu(c)=p_{\tau(c)}$ it holds that $\{c\} \in \gamma^{s}(p)$, so $u^{s}(c) \leq U^{s}(A)$. For every $\{c\} \in \gamma^{s}(\bar{p})$ such that $\mu(c)=p_{\tau(c)}^{\prime}$ it holds that $\{c\} \in \gamma^{s}\left(p^{\prime}\right)$, so $u^{s}(c) \leq U^{s}\left(A^{\prime}\right) \leq U^{s}(A)$. It follows that Condition (ii) of Definition 4.1 holds for every $s \in S_{2} \cup S_{3}$.

Step 3: Condition (iii) of Definition 4.1 holds.

For each $t \in T$, if $\bar{p}_{t}<l_{t}^{\beta(t)}$, then $p_{t}<l_{t}^{\beta(t)}$ and $p_{t}^{\prime}<l_{t}^{\beta(t)}$, so since $(p, q, A)$ and $\left(p^{\prime}, q, A^{\prime}\right)$ are both QCCEs, it follows that $q_{t}=0$.

We conclude that $(\bar{p}, q, \bar{A})$ is a QCCE.

Q.E.D.

\section{A.10 Proof of Theorem 5.16}

Since $p^{\prime} \in P(\mathcal{E}, q)$, then there is an outcome $\bar{A}$ such that $\left(p^{\prime}, q, \bar{A}\right)$ is a QCCE. Let $A^{\prime \prime}$ be defined as

$$
\begin{aligned}
A^{\prime \prime}= & A \vee \bar{A} \\
= & \left(\cup_{b \in B}\left\{(\bar{A})^{b} \mid U^{b}(A)>U^{b}(\bar{A})\right\}\right) \cup\left(\cup_{b \in B}\left\{A^{b} \mid U^{b}(A)<U^{b}(\bar{A})\right\}\right) \\
& \cup\left(\cup_{b \in B}\left\{A^{b} \mid U^{b}(A)=U^{b}(\bar{A})\right\}\right) .
\end{aligned}
$$

By Theorem 5.12, $\left(p \vee p^{\prime}, q, A^{\prime \prime}\right)$ is a QCCE. By the first part of the proof of Theorem 5.12, for every agent $i \in B \cup S$ such that $U^{i}(A)>U^{i}(\bar{A})$ or $U^{i}(A)<U^{i}(\bar{A})$, agent $i$ makes 
a trade with some other agent in both $(p, q, A)$ and $\left(p^{\prime}, q, \bar{A}\right)$, and so agent $i$ also makes a trade in $\left(p \vee p^{\prime}, q, A^{\prime \prime}\right)$. By the construction of $A^{\prime \prime}$, the set of agents who choose the no-trade option at $(p, q, A)$ is the same as the set of agents who choose the no-trade option at $\left(p \vee p^{\prime}, q, A^{\prime \prime}\right)$, i.e., $B \backslash \beta(\tau(A))=B \backslash \beta\left(\tau\left(A^{\prime \prime}\right)\right)$ and $S \backslash \sigma(\tau(A))=S \backslash \sigma\left(\tau\left(A^{\prime \prime}\right)\right)$.

Let $A^{\prime}$ be defined as

$$
\begin{aligned}
A^{\prime}= & \bar{A} \wedge A^{\prime \prime} \\
= & \left(\cup_{b \in B}\left\{(\bar{A})^{b} \mid U^{b}(\bar{A})>U^{b}\left(A^{\prime \prime}\right)\right\}\right) \cup\left(\cup_{b \in B}\left\{\left(A^{\prime \prime}\right)^{b} \mid U^{b}(\bar{A})<U^{b}\left(A^{\prime \prime}\right)\right\}\right) \\
& \cup\left(\cup_{b \in B}\left\{\left(A^{\prime \prime}\right)^{b} \mid U^{b}(\bar{A})=U^{b}\left(A^{\prime \prime}\right)\right\}\right) .
\end{aligned}
$$

By Theorem 5.12, $\left(\left(p \vee p^{\prime}\right) \wedge p^{\prime}, q, A^{\prime}\right)$ is a QCCE. By the first part of the proof of Theorem 5.12, for every agent $i \in B \cup S$ such that $U^{i}(\bar{A})>U^{i}\left(A^{\prime \prime}\right)$ or $U^{i}(\bar{A})<U^{i}\left(A^{\prime \prime}\right)$, agent $i$ makes a trade with some other agent in both $\left(p \vee p^{\prime}, q, A^{\prime \prime}\right)$ and $\left(p^{\prime}, q, \bar{A}\right)$, and so agent $i$ also makes a trade in $\left(\left(p \vee p^{\prime}\right) \wedge p^{\prime}, q, A^{\prime}\right)$. By the construction of $A^{\prime}$, the set of agents who choose the no-trade option at $\left(p \vee p^{\prime}, q, A^{\prime \prime}\right)$ is the same as the set of agents who choose the no-trade option at $\left(\left(p \vee p^{\prime}\right) \wedge p^{\prime}, q, A^{\prime}\right)$, i.e., $B \backslash \beta\left(\tau\left(A^{\prime \prime}\right)\right)=B \backslash \beta\left(\tau\left(A^{\prime}\right)\right)$ and $S \backslash \sigma\left(\tau\left(A^{\prime \prime}\right)\right)=S \backslash \sigma\left(\tau\left(A^{\prime}\right)\right)$.

Thus $B \backslash \beta(\tau(A))=B \backslash \beta\left(\tau\left(A^{\prime}\right)\right)$ and $S \backslash \sigma(\tau(A))=S \backslash \sigma\left(\tau\left(A^{\prime}\right)\right)$. The result now follows from the observation that $\left(p \vee p^{\prime}\right) \wedge p^{\prime}=p^{\prime}$.

Q.E.D.

\section{A.11 Proof of Theorem 6.4}

Let $(p, q, A)$ be a DE of $\overline{\mathcal{E}}$. For every $b \in B, \delta^{b}(p, q)=\bar{\delta}^{b}(p, q)$, so from Condition (i) of Definition 6.1, it follows that $A^{b} \in \delta^{b}(p, q)$, so Condition (i) of Definition 4.1 holds. For every $s \in S, \delta^{s}(p)=\bar{\delta}^{s}(p)$, so from Condition (ii) of Definition 6.2, it follows that $A^{s} \in \delta^{s}(p)$, so Condition (ii) of Definition 4.1 holds. Finally, for every $t \in T$ such that $p_{t}<l_{t}^{\beta(t)}$, it holds that $p_{t}<\bar{p}_{t}$, so by Condition (iv) of Definition 6.2 , we have $q_{t}=0$ and Condition (iii) of Definition 4.1 is satisfied. We have shown that $(p, q, A)$ is a QCCE of $\mathcal{E}$.

Q.E.D.

\section{References}

[1] Alkan, A., Gale, D., 1990. The core of the matching game. Games and Economic Behavior 2(3), 203-212.

[2] Andersson, T., Svensson, L.G., 2014. Non-manipulable house allocation with rent control. Econometrica 82(2), 507-539.

[3] Ausubel, L.M., Milgrom, P.R., 2002. Ascending auctions with package bidding. Advances in Theoretical Economics 1(1). 
[4] Bénassy, J. P., 1975. Neo-Keynesian disequilibrium theory in a monetary economy. The Review of Economic Studies 42(4), 503-523.

[5] Benoit, J. P., Krishna, V., 2001. Multiple-object auctions with budget constrained bidders. The Review of Economic Studies 68(1), 155-179.

[6] Candogan, O., Epitropou, M., Vohra, R., 2017. Competitive equilibirum and trading networks: a network flow approach. Working paper.

[7] Che, Y.K., Gale, I., 1998. Standard auctions with financially constrained bidders. The Review of Economic Studies 65(1), 1-21.

[8] Crawford, V.P., Knoer, E.M., 1981. Job matching with heterogeneous firms and workers. Econometrica 49(2), 437-450.

[9] Demange, G., Gale, D., 1985. The strategy structure of two-sided matching markets. Econometrica 53(4), 873-888.

[10] Demange, G., Gale, D., Sotomayor, M., 1986. Multi-item auctions. Journal of Political Economy 94(4), 863-872.

[11] Dobzinski, S., Lavi, R., Nisan, N., 2012. Multi-unit auctions with budget limits. Games and Economic Behavior 74(2), 486-503.

[12] Drèze, J. H., 1975. Existence of an exchange equilibrium under price rigidities. International Economic Review 16, 301-320.

[13] Fleiner, T., Janko, Z., Tamura, A., Teytelboym, A., 2018. Trading networks with bilateral contracts. Working paper, University of Oxford.

[14] Gale, D., Shapley, L.S., 1962. College admissions and the stability of marriage. American Mathematical Monthly 69(1), 9-15.

[15] Gul, F., Stacchetti, E., 1999. Walrasian equilibrium with gross substitutes. Journal of Economic Theory 87(1), 95-124.

[16] Hatfield, J.W., Kojima, F., 2010. Substitutes and stability for matching with contracts. Journal of Economic Theory 145, 1704-1723.

[17] Hatfield, J.W., Kominers, S.D., 2012. Matching in networks with bilateral contracts. American Economic Journal: Microeconomics 4(1), 176-208. 
[18] Hatfield, J.W., Kominers, S.D., Nichifor, A., Ostrovsky, M., Westkamp, A., 2013. Stability and competitive equilibrium in trading networks. Journal of Political Economy 121(5), 966-1005.

[19] Hatfield, J.W., Milgrom, P.R., 2005. Matching with contracts. American Economic Review 95(4), 913-935.

[20] Herings, P.J.J., 1996. Static and dynamic aspects of general disequilibrium theory. Theory and Decision Library, Series C: Game Theory, Mathematical Programming and Operations Research. Kluwer Academic Publishers, Norwell, Massachusetts.

[21] Herings, P.J.J., 2018. Equilibrium and matching under price controls. Journal of Economic Theory 177, 222-244.

[22] Illing, G., Klüh, U., 2003. Spectrum auctions and competition in telecommunications. MIT Press.

[23] Kelso, A.S., Jr., Crawford, V.P., 1982. Job matching, coalition formation, and gross substitutes. Econometrica 50, 1483-1504.

[24] Kojima, F., Sun, N., Yu, N.N., 2018. Job matching under constraints. Working paper.

[25] Koopmans, T.C., Beckmann, M., 1957. Assignment problems and the location of economic activities. Econometrica 25(1), 53-76.

[26] Kucuksenel, S., 2011. Core of the assignment game via fixed point methods. Journal of Mathematical Economics 47(1), 72-76.

[27] Laan, G. van der, Talman, A.J.J., Yang, Z., 2018. Equilibrium in the assignment market under budget constraints. Working paper.

[28] Laan, G. van der, Yang, Z., 2016. An ascending multi-item auction with financially constrained bidders. The Journal of Mechanism and Institution Design 1(1), 109-149.

[29] Liao, R.C., 2014. What drives corporate minority acquisitions around the world? The case for financial constraints. Journal of Corporate Finance 26, 78-95.

[30] Makowski, L., 1979. Value theory with personalized trading. Journal of Economic Theory 20(2), 194-212.

[31] Maskin, E.S., 2000. Auctions, development, and privatization: efficient auctions with liquidity-constrained buyers. European Economic Review 44(4), 667-681. 
[32] Ostrovsky, M., 2008. Stability in supply chain networks. American Economic Review $98(3), 897-923$.

[33] Quinzii, M., 1984. Core and competitive equilibria with indivisibilities. International Journal of Game Theory 13(1), 41-60.

[34] Roth, A. E., 1984. Stability and polarization of interests in job matching. Econometrica $52(1), 47-58$.

[35] Roth, A. E., 1986. On the allocation of residents to rural hospitals: a general property of two-sided matching markets. Econometrica 54: 425-427.

[36] Roth, A.E., Sotomayor, M.A.O., 1990. Two-sided matching: a study in game-theoretic modeling and analysis. Econometric Society Monographs No. 18. Cambridge University Press.

[37] Schlegel, J.C., 2019. Trading networks with general preferences. Working paper.

[38] Shapley, L.S., Shubik, M., 1971. The assignment game I: the core. International Journal of Game Theory 1(1), 111-130.

[39] Sun, N., Yang, Z., 2006. Equilibria and indivisibilities: gross substitutes and complements. Econometrica 74(5), 1385-1402.

[40] Talman, D., Yang, Z., 2008. A dynamic auction for differentiated items under price rigidities. Economics Letters, 99(2), 278-281.

[41] Talman, A.J.J., Yang, Z., 2015. An efficient multi-item dynamic auction with budget constrained bidders. International Journal of Game Theory, 44(3), 769-784.

[42] Younès, Y., 1975. On the role of money in the process of exchange and the existence of a non-Walrasian Equilibrium. The Review of Economic Studies 42(4), 489-501. 